\title{
Efficient Joint Estimation and Compensation of CFO, Tx/Rx Frequency-Selective I/Q Imbalance, and the MIMO Radio Channel in OFDM Systems
}

\author{
Jian Luo, ${ }^{1}$ Wilhelm Keusgen, ${ }^{2}$ and Andreas Kortke ${ }^{3}$ \\ ${ }^{1}$ Huawei Technologies Duesseldorf GmbH, European Research Center (ERC), Riesstraße 25, 80992 Munich, Germany \\ ${ }^{2}$ Fraunhofer Heinrich Hertz Institute, Einsteinufer 37, 10587 Berlin, Germany \\ ${ }^{3}$ Technische Universität Berlin, Straße des 17. Juni 135, 10623 Berlin, Germany \\ Correspondence should be addressed to Jian Luo; jianluo@huawei.com
}

Received 23 May 2012; Revised 23 October 2012; Accepted 18 November 2012

Academic Editor: Luc Vandendorpe

Copyright (C) 2013 Jian Luo et al. This is an open access article distributed under the Creative Commons Attribution License, which permits unrestricted use, distribution, and reproduction in any medium, provided the original work is properly cited.

\begin{abstract}
MIMO OFDM is a very promising technique for future wireless communication systems. By applying direct conversion architecture, low-cost, low-power, small size, and flexible implementation of MIMO OFDM systems can be realized. However, the performance of direct conversion architecture-based MIMO OFDM systems can be seriously affected by RF impairments incling carrier frequency offset (CFO) and I/Q-imbalance. While OFDM is sensitive to CFO, direct conversion architecture is sensitive to I/Q imbalance. Such RF impairments aggravate as the carrier frequency becomes higher for example, beyond $60 \mathrm{GHz}$. To achieve the desired high performance of MIMO OFDM, such RF impairments have to be compensated for. In this paper, the joint compensation of CFO, transmitter and receiver frequency-selective I/Q imbalance, and the MIMO radio channel is investigated. Two preamblebased schemes are proposed for impairment parameter estimation. The proposed preambles are constructed both in time and frequency domains and require much less overhead than the state-of-the-art designs. Furthermore, much lower computational complexity is allowed, enabling efficient implementation. The advantages and effectiveness of both proposed schemes are compared and verified by numerical simulations and complexity analysis.
\end{abstract}

\section{Introduction}

MIMO OFDM is one of the most promising techniques for achieving high data rates in future wireless communication systems. To allow low-cost, low-power, small size, and flexible implementation of MIMO OFDM systems, direct conversion architecture should be applied both at the transmitter (Tx) and the receiver (Rx). However, MIMO OFDM systems with direct conversion architecture are sensitive to RF impairments including CFO, DC-Offset, and I/Q imbalance (both at the Tx and the Rx). Since DC-Offset can be easily suppressed by AC coupling [1], we mainly focus on the investigation of $\mathrm{CFO}$ and $\mathrm{I} / \mathrm{Q}$ imbalance. While $\mathrm{CFO}$ causes common phase error and inter-carrier interference, I/Q imbalance results in mirror frequency interference. Both impairments aggravate as the carrier frequency gets higher, for example, beyond $60 \mathrm{GHz}$. Furthermore, in broadband systems, the I/Q imbalance effect is generally frequency selective, giving more challenges to the estimation and compensation.

The compensation of CFO and I/Q imbalance in SISOand MIMO OFDM systems has been studied in a number of works, for example, [2-16], respectively. Among them, $[3-5,9,12,14]$ only considered frequency-independent I/Q imbalance, which is not suitable for broadband wireless communication systems. In contrast, the other works considered frequency-selective I/Q imbalance. The work [2] has proposed a nonlinear-least-square-(NLS-) based scheme for CFO estimation and Rx-I/Q-imbalance compensation. This scheme requires exhaustive numerical search and thus, results in high computational complexity. Improvements have been made by $[6,7,11,15]$, where a closed-form linear least square (LLS) estimator and a suboptimal iterative estimator have been proposed, respectively. Both schemes have lower computational complexity than that in [2]. In 
[10], perfect CFO estimation was assumed and a per-tone equalization (PTEQ) scheme was proposed for impairment compensation. However, the PTEQ scheme suffers from slow convergence and high computational complexity. In [13], Kalman filter is applied for compensation, which can cope with fast fading MIMO channels. However, this scheme has the disadvantage of high pilot overhead (e.g., ten OFDM symbols as preamble). Many of the works above, including $[2,5,6,9,12-14]$, have the additional drawback that only $\mathrm{Rx}$ impairments were considered in the system model. Thus, if Tx-I/Q imbalance is present, the schemes of these works may suffer from severe performance degradation due to model mismatch (some performance comparisons have been shown in [7]). In [17], blind Rx-I/Q imbalance estimation and compensation schemes were proposed. When the CFO is sufficiently large, these schemes can provide good $\mathrm{Rx}-\mathrm{I} / \mathrm{Q}$ imbalance estimation even in low SNR region. The reason is that the Rx noise, which is influenced by Rx-I/Q-imbalance, is also exploited for estimation. Recently, this scheme has been extended in [18] to include Tx-I/Q-imbalance and CFO mitigation for SISO OFDM systems. For MIMO OFDM systems, this scheme needs further extension.

The most advanced state-of-the-art schemes considering $\mathrm{CFO}$ and both $\mathrm{Tx}$ - and $\mathrm{Rx}$ - frequency-selective I/Qimbalance in MIMO OFDM systems are those in [11, 15] as well as in our previous work [16]. The drawback of the schemes in $[11,15]$ is that the used preambles are not overhead and interference optimal. Moreover, the estimation schemes in $[11,15]$ require quite high computational complexity.

This paper extends our previous work [16] and presents two improved schemes for the joint estimation of CFO, Txand Rx-frequency-selective I/Q-imbalance, and the MIMO channel in OFDM systems. These improved schemes are based on a novel preamble design. Note that in this paper, we focus on indoor scenarios and assume block fading channels, for which preamble-based parameter estimation is most efficient. (When assuming block-fading channels, the MIMO channels as well as CFO and Tx- and Rx-I/Q-imbalance only need to be estimated once per communication signal frame, based on the preamble. Afterwards, these estimated parameters can be used for impairment compensation and signal equalization of the whole signal frame. However, due to residual $\mathrm{CFO}$ estimation error and phase noise, phase tracking may be necessary, which is carried out for each OFDM block.)

The novelties of the proposed schemes can be summarized as follows.

(i) The proposed preamble is used both for the estimation of CFO and Rx-I/Q-imbalance as well as the joint estimation of Tx-I/Q-imbalance and the MIMO channel. In contrast, most existing schemes apply different preambles for these two estimation procedures (e.g., $[6,7,11,15])$. Thus, compared to the preambles used in the reference works, our preamble is much more overhead efficient.

(ii) When designing the preamble, the orthogonality between different $\mathrm{Tx}$ antennas as well as between direct and image channels (see [19]) is taken into account, allowing better estimation accuracy than $[11,15]$.

(iii) The proposed preambles have low crest factor, which enables "preamble power boosting."

(iv) The proposed estimation schemes require much lower computational complexity than those in $[11,15]$.

(v) As most of the existing schemes, the CFO estimation and $\mathrm{Rx}-\mathrm{I} / \mathrm{Q}$-imbalance estimation/compensation of proposed schemes rely on the negative phase rotation (the direction of the phase rotation (caused by $\mathrm{CFO}$ ) without Rx-I/Q-imbalance is referred to as "positive") in the mirror frequency interference signal caused by $\mathrm{Rx}-\mathrm{I} / \mathrm{Q}$-imbalance. As a result, small CFOs can cause difficulties for both estimation and compensation. Conventionally, for example, in [6, 7], "hard switching" is applied, that is, CFO values smaller than a threshold are regarded as zero (correspondingly, another I/Q-imbalance and channel estimation schemes will be applied, which are suitable for the cases without CFO). However, an improperly chosen threshold can cause considerable performance degradation. To solve this problem, we have proposed soft metrics which change adaptively with the SNR and allow "soft switching."

The advantages of the proposed schemes are verified both by numerical simulation and complexity analysis. Compared to our previous work [16], the extension includes a new scheme and much more extensive simulation results and analysis. Based on the above descriptions, the proposed schemes outperform the state-of-the-art schemes and provide very promising options for joint $\mathrm{CFO}, \mathrm{Tx}-/ \mathrm{Rx} \mathrm{I} / \mathrm{Q}$ imbalance and channel estimation in indoor MIMO OFDM systems.

This paper is organized as follows. Section 2 describes the system model and the equivalent baseband models. Section 3 describes the joint compensation structure and the calculation of compensation coefficients. Sections 4 and 5 describe two preamble-based parameter estimation schemes. Section 6 shows the simulation results. Section 7 provides complexity analysis. Section 8 concludes this paper.

\section{Signal and System Model}

Figure 1 shows the MIMO system model considering both $\mathrm{CFO}$ and $\mathrm{Tx} / \mathrm{Rx}$ frequency-selective I/Q-imbalance. The numbers of Tx and Rx antennas are $N_{T}$ and $N_{R}$, respectively. The amplitude and phase imbalance at the $i$ th modulator or at the $r$ th demodulator are indicated by $g_{T ; R}^{(i)\langle\langle r}$ and $\varphi_{T ; R}^{(i) ;\langle r\rangle}$, respectively. The impulse response of Tx or $\mathrm{Rx}$ low-pass Filters (LPF) in the I and Q branches are indicated by $h_{\mathrm{TI} ; \mathrm{RI}}^{(i)\langle r}(t)$ and $h_{\mathrm{TQ} ; \mathrm{RQ}}^{(i) ;(t)}(t)$, respectively. All different Tx/Rx branches have different I/Q-imbalance parameters as well as different LPF impulse response. The ordinary carrier frequency is $f_{c}$, while a CFO, $\Delta f$, is present at all $\mathrm{Rx}$ demodulators. The impulse response of the RF components at the $i$ th Tx or the $r$ th Rx are modeled as $h_{\mathrm{RF}, \mathrm{Tx} ; \mathrm{Rx}}^{(i) ;\langle\mathrm{r}}(t)$. The radio channel between the $i$ th $\mathrm{Tx}$ 


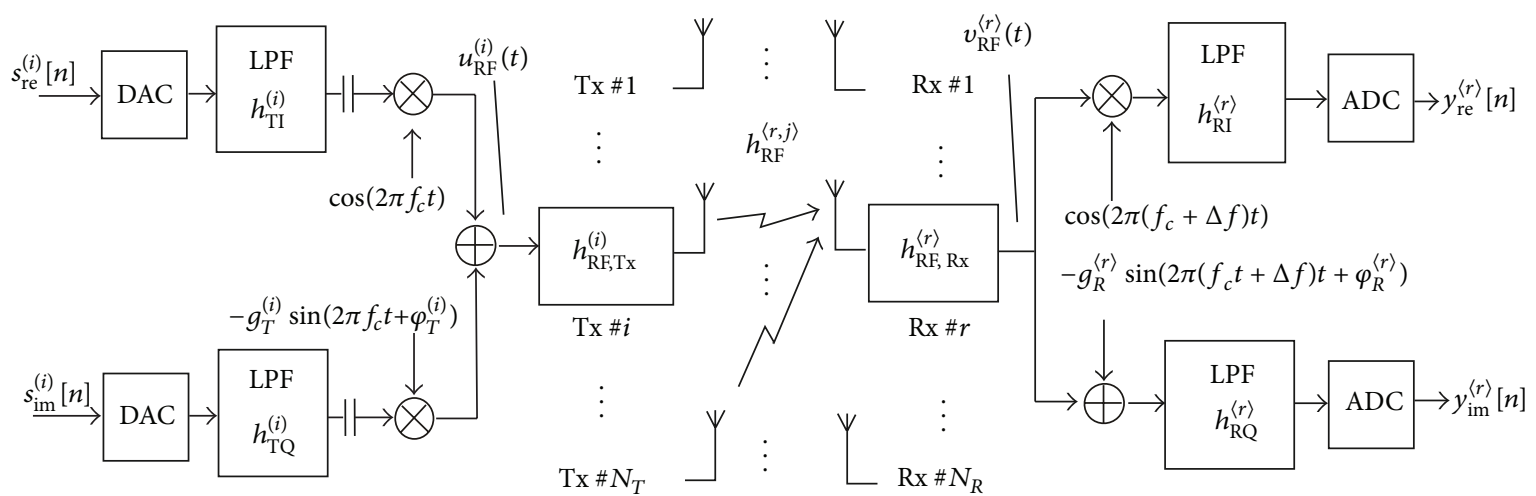

FIGURE 1: System model with CFO and frequency-selective I/Q imbalance.

and the $r$ th $\mathrm{Rx}$ is modeled by $h_{\mathrm{RF}}^{\langle r, i\rangle}(t)$, which is assumed to be quasi static (block fading).

For further analysis, an equivalent baseband model should be derived. From Figure 1, the relation between the LPF inputs at Tx, $s_{\text {re }}^{(i)}(t)$ and $s_{\text {im }}^{(i)}(t)$, and the modulated signal $u_{\mathrm{RF}}^{(i)}(t)$ (shown in Figure 1) can be expressed as

$$
\begin{aligned}
u_{\mathrm{RF}}(t)= & \left(s_{\mathrm{re}}(t) * h_{\mathrm{TI}}(t)\right) \cos \left(2 \pi f_{c} t\right) \\
& -\left(s_{\mathrm{im}}(t) * h_{\mathrm{TQ}}(t)\right) g_{T} \sin \left(2 \pi f_{c} t+\varphi_{T}\right) \\
= & \operatorname{Re}\{\underbrace{\left(s(t) * h_{\mathrm{D}, \mathrm{T}}(t)+s^{*}(t) * h_{\mathrm{I}, \mathrm{T}}(t)\right)}_{u(t)} e^{j 2 \pi f_{c} t}\},
\end{aligned}
$$

where $s(t)=s_{\text {re }}(t)+j s_{\text {im }}(t)$ and $h_{\mathrm{D} ; \mathrm{I}, \mathrm{T}}(t):=\left(h_{\mathrm{TI}}(t) \pm\right.$ $\left.g_{T} e^{j \varphi_{T}} h_{\mathrm{TQ}}(t)\right) / 2$ with the Tx index $i$ omitted for simplicity. Note that $u(t)$ is the complex envelope of $u^{\mathrm{RF}}(t)$. Let the demodulator input signal be expressed as $v_{\mathrm{RF}}(t)=$ $\mathscr{R} e\left\{v(t) e^{j 2 \pi f_{c} t}\right\}$ (shown in Figure 1), where the $\mathrm{Rx}$ index $r$ is omitted for simplicity. The relation between the complex envelope $v(t)$ and the I/Q ADC input $y(t)=y_{\mathrm{re}}(t)+j y_{\mathrm{im}}(t)$ can be expressed as

$$
y(t)=\left(v(t) e^{-j 2 \pi \Delta f t}\right) * h_{\mathrm{D}, \mathrm{R}}(t)+\left(v(t) e^{-j 2 \pi \Delta f t}\right)^{*} * h_{\mathrm{I}, \mathrm{R}}(t),
$$

where $h_{\mathrm{D} ; \mathrm{I}, \mathrm{R}}(t):=\left(h_{\mathrm{RI}}(t) \pm g_{R} e^{\mp j \varphi_{R}} h_{\mathrm{RQ}}(t)\right) / 2$.

Based on (1) and (2), the equivalent discrete time baseband model in Figure 2 can be obtained, where $h_{\mathrm{D} ; \mathrm{I}, \mathrm{T}}^{(i)}[n]$ and $h_{\mathrm{D} ; \mathrm{I}, \mathrm{R}}^{\langle r\rangle}[n]$ are the discrete versions of $h_{\mathrm{D} ; \mathrm{I}, \mathrm{T}}^{(i)}(t)$ and $h_{\mathrm{D} ; \mathrm{I}, \mathrm{R}}^{\langle r\rangle}(t)$, respectively. Moreover, $h^{\langle r, i\rangle}[n]$ is the equivalent baseband channel between the ith $\mathrm{Tx}$ and the $r$ th $\mathrm{Rx}$ and includes the effects of $h_{\mathrm{RF}}^{\langle r, i\rangle}(t), h_{\mathrm{RF}, \mathrm{Tx}}^{(i)}(t)$, and $h_{\mathrm{RF}, \mathrm{Rx}}^{\langle r\rangle}(t)$ in Figure 1. The CFO influence is modeled by the multiplication with $e^{j(2 \pi \epsilon n / N)}$, where $\epsilon=-\Delta f N / f_{s}$ with $f_{s}$ as the sampling frequency (e.g., Nyquist sampling frequency). Finally, $\eta^{\langle r\rangle}[n]$ is the additive white Gaussian noise (AWGN) at the $r$ th $\mathrm{Rx}$ branch (equivalent discrete version of the RF frontend noise, but before LPF filtering) with $E\left\{\left|\eta^{\langle r\rangle}[n]\right|^{2}\right\}=\sigma_{\mathrm{n}}^{2}$.
To further simplify the baseband model, we observe the following relation:

$$
\begin{aligned}
(s & {\left.[n] e^{j(2 \pi / N) \epsilon n}\right) * h[n] } \\
& =\sum_{m=-\infty}^{\infty} h[m]\left(s[n-m] e^{j(2 \pi / N) \epsilon(n-m)}\right) \\
& =\left(\sum_{m=-\infty}^{\infty} h[m] e^{-j(2 \pi / N) \epsilon m} s[n-m]\right) e^{j(2 \pi / N) \epsilon n} \\
& =\left[s[n] *\left(h[n] e^{-j(2 \pi / N) \epsilon n}\right)\right] e^{j(2 \pi / N) \epsilon n},
\end{aligned}
$$

where $s[n]$ and $h[n]$ represent an arbitrary input sequence and an FIR filter, respectively. Equation (3) implies the equivalent system structures in Figure 3, where the following notation is used throughout this paper (similar to [6]):

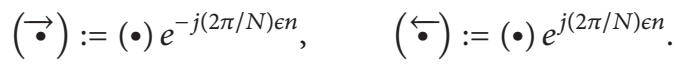

By applying the equivalent structure of Figure 3 to the input-output relation of the baseband model in Figure 2, we obtain the following expression:

$$
\begin{aligned}
y^{\langle r\rangle}[n]= & x_{\mathrm{D}}^{\langle r\rangle}[n] e^{j(2 \pi \epsilon n / N)}+x_{\mathrm{I}}^{\langle r\rangle}[n] e^{-j(2 \pi \epsilon n / N)}+\widetilde{\eta}^{\langle r\rangle}[n], \\
x_{\mathrm{D}}^{\langle r\rangle}[n]:= & \sum_{i=1}^{N_{T}} s^{(i)}[n] * h_{1}^{\langle r, i\rangle}[n]+s^{(i) *}[n] * h_{2}^{\langle r, i\rangle}[n], \\
x_{\mathrm{I}}^{\langle r\rangle}[n]:= & \sum_{i=1}^{N_{T}} s^{(i)}[n] * h_{3}^{\langle r, i\rangle}[n]+s^{(i) *}[n] * h_{4}^{\langle r, i\rangle}[n], \\
\tilde{\eta}^{\langle r\rangle}[n]:= & \eta^{\langle r\rangle}[n] e^{j(2 \pi \epsilon n / N)} h_{\mathrm{D}, \mathrm{R}}^{\langle r\rangle}[n] \\
& +\eta^{\langle r\rangle *}[n] e^{-j(2 \pi \epsilon n / N)} h_{\mathrm{I}, \mathrm{R}}^{\langle r\rangle}[n],
\end{aligned}
$$

with

$$
\begin{aligned}
& h_{1 ; 2}^{\langle r, i\rangle}[n]:=h_{\mathrm{D} ; \mathrm{I}, \mathrm{T}}^{(i)}[n] * h^{\langle r, i\rangle}[n] * \overrightarrow{h_{\mathrm{D}, \mathrm{R}}^{\langle r\rangle}[n]}, \\
& h_{3 ; 4}^{\langle r, i\rangle}[n]:=h_{\mathrm{I} ; \mathrm{D}, \mathrm{T}}^{(i) *}[n] * h^{\langle r, i\rangle *}[n] * \overleftarrow{h_{\mathrm{I}, \mathrm{R}}^{\langle r\rangle}[n]}
\end{aligned}
$$




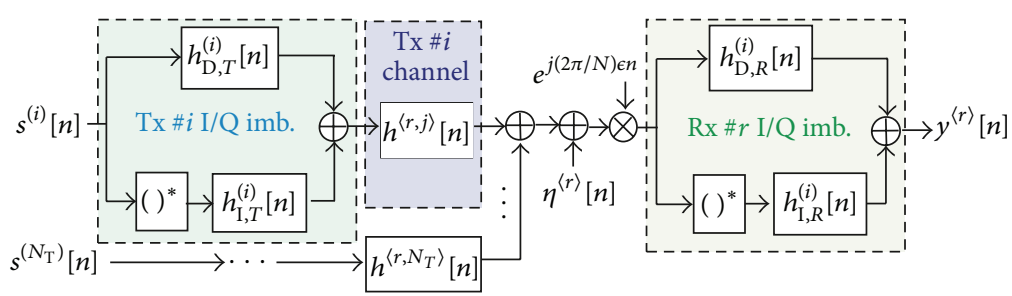

FIGURE 2: Equivalent baseband model with CFO and I/Q imbalance.

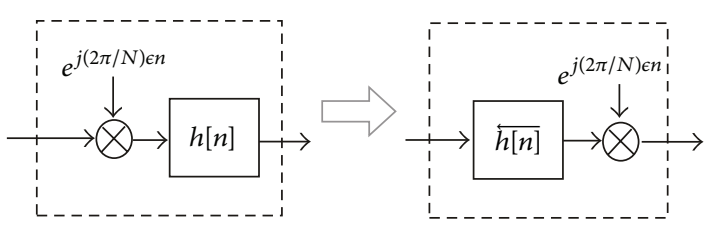

FIGURE 3: Exchanging the positions of a phase rotator and an FIR filter.

where $h_{1}^{\langle r, i\rangle} \sim h_{4}^{\langle r, i\rangle}$ are assumed to be of length $L_{\mathrm{h}}$ FIR filters. Equation (5) yields the simplified baseband model in Figure 4.

Finally, we remark that although the model of Figure 2 can be found in similar mathematical expression in the literature, for example, $[11,15]$, the model of Figure 4 is novel (to the best of the authors' knowledge).

\section{Compensation of CFO, Frequency-Selective I/Q-Imbalance, and the MIMO Channel}

For the compensation of CFO, frequency-selective I/Qimbalance, and the MIMO channel, an extended version of the hybrid domain compensation structure in [7] is applied (similar to [11, 15]), which is shown in Figure 5. Within each $\mathrm{Rx}$ branch, Rx-I/Q-imbalance and CFO are compensated in time domain. Afterwards, Tx-I/Q-imbalance and the MIMO channels are compensated in frequency domain.

From Figure 5, the $\mathrm{Rx}$ signal after $\mathrm{Rx}-\mathrm{I} / \mathrm{Q}$-imbalance compensation can be expressed as

$$
\begin{aligned}
y^{\langle r\rangle}[n]= & y_{\mathrm{re}}^{\langle r\rangle}\left[n-n_{\tau}\right] \\
& +j\left(c y_{\mathrm{re}}^{\langle r\rangle}\left[n-n_{\tau}\right]+h^{C\langle r\rangle}[n] * y_{\mathrm{im}}^{\langle r\rangle}[n]\right),
\end{aligned}
$$

where $n_{\tau}$ is the dominant-tap index (we assume the index starts from 0 ) of the FIR filter $h^{C\langle r\rangle}[n]$, which is of length $L_{c}$. Equation (7) can be equivalently written as

$$
\dot{y}^{\langle r\rangle}[n]=w_{\mathrm{D}}^{\langle r\rangle}[n] * y^{\langle r\rangle}[n]+w_{\mathrm{I}}^{\langle r\rangle}[n] * y^{\langle r\rangle *}[n],
$$

with

$$
w_{\mathrm{D} ; \mathrm{I}}^{\langle r\rangle}[n]=\frac{1}{2}\left(\delta\left[n-n_{\tau}\right]\left(1+j c^{\langle r\rangle}\right) \pm h^{C\langle r\rangle}[n]\right)
$$

where $\delta[n]$ is the discrete time impulse function. By substituting (5) into (8), we have

$$
\begin{aligned}
& \dot{y}^{\langle r\rangle}[n]=e^{j(2 \pi \epsilon n / N)} \\
& \times \sum_{i=1}^{N_{T}}\left[s^{(i)}[n] * h_{\mathrm{D}}^{E\langle r, i\rangle}[n]+s^{(i) *}[n] * h_{\mathrm{I}}^{E\langle r, i\rangle}[n]\right] \\
& +e^{-j(2 \pi \epsilon n / N)} \\
& \times \sum_{i=1}^{N_{T}}\left[s^{(i)}[n] * \vartheta_{\mathrm{D}}^{E\langle r, i\rangle}[n]+s^{(i) *}[n] * \vartheta_{\mathrm{I}}^{E\langle r, i\rangle}[n]\right] \\
& +\dot{\eta}^{\langle r\rangle}[n] \text {, } \\
& h_{\mathrm{D} ; \mathrm{I}}^{E\langle r, i\rangle}[n]:=h_{1 ; 2}^{\langle r, i\rangle}[n] * \overrightarrow{w_{\mathrm{D}}^{\langle r\rangle}[n]} \\
& +h_{4 ; 3}^{\langle r, i\rangle *}[n] * \overrightarrow{w_{\mathrm{I}}^{\langle r\rangle}[n]}, \\
& \vartheta_{\mathrm{D} ; \mathrm{I}}^{E\langle r, i\rangle}[n]:=h_{3 ; 4}^{\langle r, i\rangle}[n] * \overleftarrow{w_{\mathrm{D}}^{\langle r\rangle}[n]} \\
& +h_{2 ; 1}^{\langle r, i\rangle *}[n] * \overleftarrow{w_{\mathrm{I}}^{\langle r\rangle}[n]}
\end{aligned}
$$

where $\dot{\eta}^{\langle r\rangle}[n]$ is the noise after Rx-I/Q-imbalance compensation. From Figure 2, we can see that the phase rotation $e^{-j(2 \pi \epsilon n / N)}$ in (5) and (10) is caused by the Rx-I/Q-imbalance. Thus, after Rx-I/Q-imbalance compensation, all signal components in (10) having the $e^{-j(2 \pi \epsilon n / N)}$ phase rotation should be eliminated, that is,

$$
\vartheta_{\mathrm{D}}^{E\langle r, i\rangle}[n]=0, \quad \vartheta_{\mathrm{I}}^{E\langle r, i\rangle}[n]=0, \quad \forall i .
$$

With (6) and (9), it can be proved that the two equations in (12) are equivalent. We will show in Section 4.3 that from (11), (12), and the estimates of $\epsilon$ and $h_{1}^{\langle r, i\rangle}[n] \sim h_{4}^{\langle r, i\rangle}[n]$, the Rx-I/Q-imbalance compensation coefficients $c^{\langle r\rangle}$ and $h^{C\langle r\rangle}[n]$, for all $r$, can be computed. 


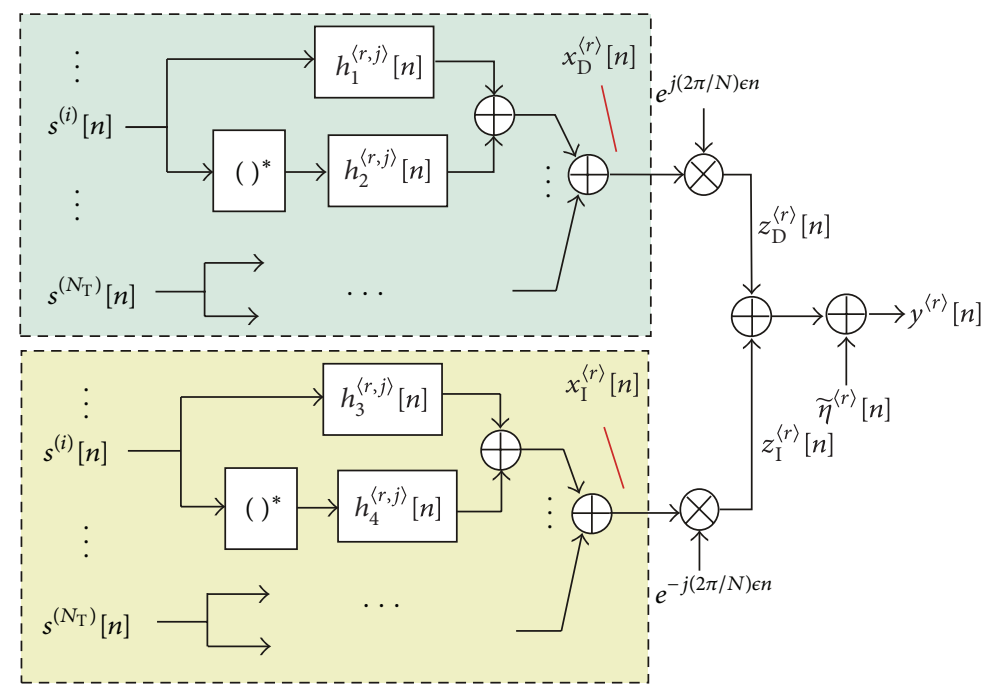

FIGURE 4: Simplified baseband model with CFO and frequency-selective I/Q imbalance.

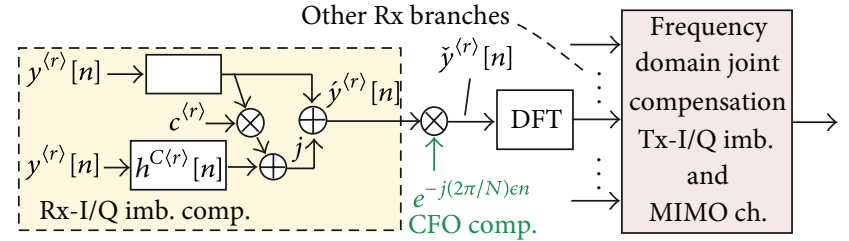

FIgURE 5: Hybrid domain compensation structure.

After successful compensation of Rx-I/Q-imbalance and CFO, the signal $\check{y}^{\langle r\rangle}[n]$ in Figure 5 can be expressed as

$$
\begin{aligned}
\check{y}^{\langle r\rangle}[n]= & \sum_{i=1}^{N_{T}}\left(s^{(i)}[n] * h_{\mathrm{D}}^{E\langle r, i\rangle}[n]+s^{(i) *}[n] * h_{\mathrm{I}}^{E\langle r, i\rangle}[n]\right) \\
& +\check{\eta}^{\langle r\rangle}[n],
\end{aligned}
$$

where $\check{\eta}^{\langle r\rangle}[n]$ is the corresponding noise term.

Let $s_{b}^{(i)}[n]$, for all $n \in[0, N-1]$, be the $b$ th transmitted OFDM symbol at the $i$ th Tx antenna, and let $\check{y}_{b}^{\langle r\rangle}[n]$, for all $n \in[0, N-1]$, be the corresponding received OFDM symbol after Rx-I/Q-imbalance and CFO compensation. Their DFTs are indicated by $S_{b}^{(i)}[k]$ and $\check{Y}_{b}^{\langle r\rangle}[k]$, respectively. Assuming sufficient cyclic prefix (CP) length $N_{\mathrm{CP}}$, (13) yields

$$
\begin{aligned}
\check{Y}_{b}^{\langle r\rangle}[k]= & \sum_{i=1}^{N_{T}}\left(S_{b}^{(i)}[k] H_{\mathrm{D}}^{E\langle r, i\rangle}[k]+S_{b}^{(i) *}[-k] H_{\mathrm{I}}^{E\langle r, i\rangle}[k]\right) \\
& +\check{N}^{\langle r\rangle}[k],
\end{aligned}
$$

where $H_{\mathrm{D} ; \mathrm{I}}^{E\langle r, i\rangle}[k]=\mathscr{F}_{N}\left\{h_{\mathrm{D} ; \mathrm{I}}^{E\langle r, i\rangle}[n]\right\}$. Equation (14) can be rewritten in different matrix equations for all possible antenna diversity or spatial multiplexing schemes according to [19]. Based on these matrix equations, various equalization techniques, for example, zero forcing and MMSE, can be used to recover the original data symbols. In the following, we will show how to obtain the compensation coefficients based on novel preamble designs.

\section{Joint Estimation Scheme 1: Closed-Form CFO Estimation-Based Method}

This scheme is developed based on the baseband model of Figure 4. First, a special preamble is applied to estimate $\epsilon$ and $h_{1}^{\langle r, i\rangle}[n] \sim h_{4}^{\langle r, i\rangle}[n]$. Based on the estimates of $\epsilon$ and $h_{1}^{\langle r, i\rangle}[n] \sim$ $h_{4}^{\langle r, i\rangle}[n]$, all required coefficients for the compensation of CFO and I/Q-imbalance can be calculated.

4.1. Preamble Design. Inspired by [5], the proposed preamble consists of a 3 -fold repetition of a basic sequence $\dot{s}^{(i)}[n], n=$ $0, \ldots, N_{P}-1$, which is constructed according to the frequency domain separation (FDS) design in [19] and varies for different Tx antennas. To distinguish the preamble from the data signals, all signals in Figure 4 are extended with the notation $(\cdot)$. The 3 -fold repetition structure is used to estimate $\epsilon, \dot{x}_{\mathrm{D}}^{\langle r\rangle}[n]$, and $\dot{x}_{\mathrm{I}}^{\langle r\rangle}[n]$ in Figure 4. Based on $\dot{x}_{\mathrm{D}}^{\langle r\rangle}[n]$ and $\dot{s}^{(i)}[n], h_{1}^{\langle r, i\rangle}[n]$ and $h_{2}^{\langle r, i\rangle}[n]$ can be estimated, while based on $\dot{x}_{\mathrm{I}}^{\langle r\rangle}[n]$ and $\dot{s}^{(i)}[n], h_{3}^{\langle r, i\rangle}[n]$ and $h_{4}^{\langle r, i\rangle}[n]$ can be estimated.

Since 3 -fold repetition is applied, we should minimize the length of the basic sequence, $N_{P}$, to minimize the preamble overhead. To apply the FDS design in [19], $\dot{s}^{(i)}[n]$ is constructed as an OFDM symbol with $N_{P}$ subcarriers, where $N_{P}$ could be different from the number of subcarriers in the data OFDM symbols, $N$. Let $\dot{S}^{(i)}[k]=\mathscr{F}_{N_{P}}\left\{\dot{s}^{(i)}[n]\right\}$. As mentioned above, $\dot{s}^{(i)}[n]$ is used to estimate $2 N_{T}$ equivalent channel impulse response of length $L_{\mathrm{h}}$ (i.e., $h_{1 ; 3}^{\langle r, i\rangle}[n]$ and $\left.h_{2 ; 4}^{\langle r, i\rangle}[n]\right)$. Thus, at least $2 N_{T} L_{\mathrm{h}}$ subcarriers should be active within $\dot{S}^{(i)}[k]$. In order to reserve the same guardband as the OFDM data symbols, we should have $N_{P} \geq 2 N_{T} L_{\mathrm{h}} N / N_{D}$. 
To facilitate the FFT implementation, we choose $N_{P}=$ $2^{\left[\log _{2}\left(2 N_{T} L_{\mathrm{h}} N / N_{D}+N_{\mathrm{a}}\right)\right]}$, where $N_{\mathrm{a}}$ is a number to adjust $N_{P}$. The number of null subcarriers in the basic sequence is $N_{0, P}=$ $\left\lceil N_{P} N / N_{D}\right\rceil$, while the number of active subcarriers is $N_{D, P}=$ $N_{P}-N_{0, P}$. Now, the active subcarriers can be allocated to different Tx antennas according to the FDS design in [19]. We denote by $\mathscr{I}_{i}$ in $\left|\mathscr{I}_{i}\right|=L^{\prime}$ the index set of the allocated subcarriers for the ith Tx. According to the FDS design in [19], the index sets $\mathscr{I}_{i}$, for all $i$ should fulfill the following interference avoidance requirements:

(1) $\mathscr{I}_{i}$, for all $i$, is an equidistant subcarrier set, so that intertap interference of the estimated channel impulse response can be avoided,

(2) $\mathscr{I}_{i_{0}} \cap \mathscr{I}_{i_{1}}=\emptyset$, for all $i_{0} \neq i_{1}$, to avoid inter-Tx-antenna interference,

(3) $\mathscr{I}_{i_{0}} \cap\left(-\mathscr{I}_{i_{1}}\right)=\emptyset$, for all $i_{0}, i_{1}$, to avoid the interference between direct and image channels.

By assigning values of a length of $L^{\prime}$ constant amplitude zero auto correlation (CAZAC) sequence $T^{(i)}(\kappa)$ with $\left|T^{(i)}(\kappa)\right|=1$ to the allocated subcarriers of each basic sequence, that is,

$$
\begin{gathered}
\dot{S}^{(i)}\left[\left(\mathscr{I}_{i}\right)_{\kappa}\right]=T^{(i)}(\kappa), \quad \forall \kappa=0, \ldots, L^{\prime}-1, \\
\dot{S}^{(i)}[k]=0, \quad \forall k \notin \mathscr{I}_{i},
\end{gathered}
$$

low crest factor of the basic sequence can be achieved. (For a CAZAC sequence, the crest factor remains constant after DFT or IDFT. Assigning the values of a CAZAC sequence to partially equidistant subcarriers with distance $d$ can be interpreted as a $d$-fold repetition, phase rotation (constant phase difference between neighboring samples) and fractional oversampling (due to guardband reservation) of the IDFT of the original CAZAC sequence. All these operations have negligible effect on the crest factor. Note that not the crest factor of the discrete sequence but of the corresponding analog baseband signal is used as criterion.) Finally, the total length of the preamble is $N_{\mathrm{CP}}+3 N_{P}$.

\subsection{Estimation Scheme}

4.2.1. Closed-Form Estimation of CFO. Let $\dot{y}_{b}^{\langle r\rangle}[n]$ be the received signal of the $b$ th repetition of the basic sequence. Equation (5) yields the following expression:

$$
\begin{aligned}
& \dot{y}_{b}^{\langle r\rangle}[n]= \dot{z}_{\mathrm{D}}^{\langle r\rangle}[n] e^{j\left(2 \pi \epsilon N_{P}(b-1) / N\right)} \\
&+\dot{z}_{\mathrm{I}}^{\langle r\rangle}[n] e^{-j\left(2 \pi \epsilon N_{P}(b-1) / N\right)}+\dot{\tilde{\eta}}_{b}^{\langle r\rangle}[n], \\
& \forall n=0, \ldots, N_{P}-1,
\end{aligned}
$$

with $\dot{z}_{\mathrm{D}}^{\langle r\rangle}[n]:=\dot{x}_{\mathrm{D}}^{\langle r\rangle}[n] e^{j(2 \pi \epsilon n / N)}$,

$$
\dot{z}_{\mathrm{I}}^{\langle r\rangle}[n]:=\dot{x}_{\mathrm{I}}^{\langle r\rangle}[n] e^{-j(2 \pi \epsilon n / N)} .
$$

With $b=1,2,3$, (16) yields 3 equations. According to [5], if we ignore the noise term, the following matrix equation can be obtained:

$$
\dot{\mathbf{y}}_{2} 2 \cos \Omega=\dot{\mathbf{y}}_{13}
$$

where

$$
\begin{aligned}
& \Omega:=\frac{2 \pi \epsilon N_{P}}{N}, \\
& \dot{\mathbf{y}}_{2}:=\left[\left(\dot{\mathbf{y}}_{2}^{\langle 1\rangle}\right)^{T}, \ldots,\left(\dot{\mathbf{y}}_{2}^{\left\langle N_{R}\right\rangle}\right)^{T}\right]^{T}, \\
& \dot{\mathbf{y}}_{13}:=\left[\left(\dot{\mathbf{y}}_{1}^{\langle 1\rangle}+\dot{\mathbf{y}}_{3}^{\langle 1\rangle}\right)^{T}, \ldots,\left(\dot{\mathbf{y}}_{1}^{\left\langle N_{R}\right\rangle}+\dot{\mathbf{y}}_{3}^{\left\langle N_{R}\right\rangle}\right)^{T}\right]^{T}, \\
& \dot{\mathbf{y}}_{b}^{\langle r\rangle}:=\left[\dot{y}_{b}^{\langle r\rangle}[0], \ldots, \dot{y}_{b}^{\langle r\rangle}\left[N_{P}-1\right]\right]^{T} .
\end{aligned}
$$

The least-square estimation (LSE) of $\cos \Omega$ is

$$
\widehat{\cos \Omega}=\frac{1}{2} \mathscr{R} e\left\{\left(\dot{\mathbf{y}}_{2}\right)^{\dagger} \dot{\mathbf{y}}_{13}\right\}=\frac{1}{2\left\|\dot{\mathbf{y}}_{2}\right\|_{2}^{2}} \mathscr{R} e\left\{\left(\dot{\mathbf{y}}_{2}\right)^{H} \dot{\mathbf{y}}_{13}\right\},
$$

which yields the following closed-form estimator (CLFE):

$$
\widehat{\epsilon}= \pm \frac{N}{2 \pi N_{P}} \cos ^{-1}(\widehat{\cos \Omega}) .
$$

Note that (20) is the extension of the estimator in [5, (Equation (15)] to exploit the $\mathrm{Rx}$ array gain and diversity gain. The sign ambiguity in (19) can be solved by taking the sign of the following rough CFO estimator:

$$
\widehat{\epsilon}^{\text {rough }}=\frac{N}{2 \pi N_{P}} \arg \left\{\left[\dot{\mathbf{y}}_{1}^{T}, \dot{\mathbf{y}}_{2}^{T}\right]^{*}\left[\dot{\mathbf{y}}_{2}^{T}, \dot{\mathbf{y}}_{3}^{T}\right]^{T}\right\} .
$$

4.2.2. Separation of $\dot{x}_{\mathrm{D}}^{\langle r\rangle}[n]$ and $\dot{x}_{\mathrm{I}}^{\langle r\rangle}[n]$. To obtain estimates of $\dot{x}_{\mathrm{D} ; \mathrm{I}}^{\langle r\rangle}[n], \dot{z}_{\mathrm{D}}^{\langle r\rangle}[n]$ and $\dot{z}_{\mathrm{I}}^{\langle r\rangle}[n]$ should be obtained first. For this purpose, we rewrite (16) into the following matrix form:

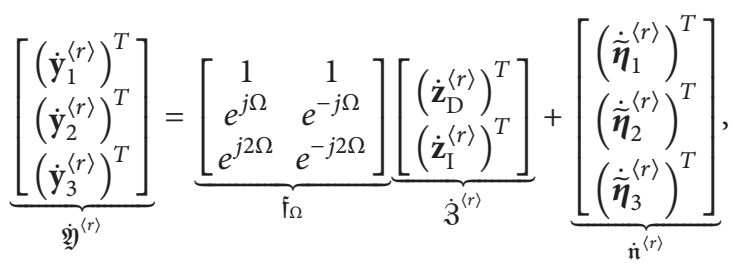

where $\dot{\mathbf{z}}_{\mathrm{D} ; \mathrm{I}}^{\langle r\rangle}=\left[\dot{z}_{\mathrm{D} ; \mathrm{I}}^{\langle r\rangle}[0], \ldots, \dot{z}_{\mathrm{D} ; \mathrm{I}}^{\langle r\rangle}\left[N_{P}-1\right]\right]^{T}$ and $\dot{\tilde{\boldsymbol{\eta}}}_{b}^{\langle r\rangle}=$ $\left[\dot{\tilde{\eta}}_{b}^{\langle r\rangle}[0], \ldots, \dot{\tilde{\eta}}_{b}^{\langle r\rangle}\left[N_{P}-1\right]\right]^{T}$.

Now, $\dot{\mathfrak{X}}^{\langle r\rangle}$ can be estimated by

$$
\widehat{\dot{\mathfrak{Z}}}^{\langle r\rangle}=\mathbf{A}^{\langle r\rangle} \dot{\mathfrak{Y}}^{\langle r\rangle}
$$

where $\mathbf{A}^{\langle r\rangle}$ is a $2 \times 3$ matrix and can be obtained by applying the following two different criteria.

(1) Minimization of the cost function $\left\|\dot{3}^{\langle r\rangle}-\mathbf{A}^{\langle r\rangle} \dot{\mathfrak{Y}}^{\langle r\rangle}\right\|_{F}^{2}$. In this case, we have

$$
\mathbf{A}^{\langle r\rangle}=\left(\mathfrak{f}_{\Omega}\right)^{\dagger}, \quad \forall r
$$

which corresponds to an LSE (this is true only when $\Omega$ is perfectly known). 
(2) Minimization of the cost function $E\left\{\left\|\dot{\boldsymbol{Z}}^{\langle r\rangle}-\mathbf{A}^{\langle r\rangle} \dot{\mathfrak{Y}}^{\langle r\rangle}\right\|_{F}^{2}\right\}$. In this case, we have

$$
\mathbf{A}^{\langle r\rangle}=\mathfrak{D}^{\langle r\rangle} \mathfrak{f}_{\Omega}^{H}\left(\mathfrak{f}_{\Omega} \mathfrak{D}^{\langle r\rangle} \mathfrak{f}_{\Omega}^{H}+\frac{1}{\rho^{\langle r\rangle}} \mathbf{I}_{3}\right)^{-1},
$$

where $\rho^{\langle r\rangle}$ is the Rx SNR at the $r$ th Rx antenna and $\mathfrak{D}^{\langle r\rangle}=$ $\operatorname{diag}\left\{\left[P_{\mathrm{D}}^{\langle r\rangle} / P^{\langle r\rangle}, P_{\mathrm{I}}^{\langle r\rangle} / P^{\langle r\rangle}\right]\right\}$ with $P_{\mathrm{D} ; \mathrm{I}}^{\langle r\rangle}=\left(1 / N_{P}\right) E\left\{\left(\dot{\mathbf{z}}_{\mathrm{D} ; \mathrm{I}}^{\langle r\rangle}\right)^{H} \dot{\mathbf{z}}_{\mathrm{D} ; \mathrm{I}}^{\langle r\rangle}\right\}$ and $P^{\langle r\rangle}=P_{\mathrm{D}}^{\langle r\rangle}+P_{\mathrm{I}}^{\langle r\rangle}$. By assuming an approximate value $\widehat{v}$ for the $\mathrm{Rx}$ image rejection ratio (IRR) $v^{\langle r\rangle}:=$ $P_{\mathrm{D}}^{\langle r\rangle} / P_{\mathrm{I}}^{\langle r\rangle}$ at all $\mathrm{Rx}$ branches (the $\mathrm{Rx}$ IRR is defined as $\sum_{n=0}^{L_{\mathrm{h}}-1}\left|h_{\mathrm{D}, \mathrm{R}}^{\langle r\rangle}[n]\right|^{2} / \sum_{n=0}^{L_{\mathrm{h}}-1}\left|h_{\mathrm{I}, \mathrm{R}}^{\langle r\rangle}[n]\right|^{2}$ and reflects the relation between the desired signal and the mirror)), we can approximate $\mathfrak{D}^{\langle r\rangle}$ with $\mathfrak{D}=\operatorname{diag}\{[\widehat{v} /(\widehat{v}+1), 1 /(\widehat{v}+1)]\}$, for all $r$. Thus, $\mathbf{A}^{\langle r\rangle}$ becomes independent of $r$. The estimation using (25) corresponds to a linear minimum mean square error (LMMSE).

In Section 6, the performance of the two estimators above will be compared based on simulation results. Now, with $\widehat{\dot{3}}^{\langle r\rangle}$ and $\widehat{\epsilon}$, we can easily obtain estimates of $\dot{x}_{\mathrm{D} ; \mathrm{I}}^{\langle r\rangle}[n]$ from (16).

4.2.3. Estimation of $h_{1}^{\langle r, i\rangle}[n] \sim h_{4}^{\langle r, i\rangle}[n]$. Let $\dot{\mathbf{x}}_{\mathrm{D} ; \mathrm{I}}^{\langle r\rangle}=$ $\left[\dot{x}_{\mathrm{D} ; \mathrm{I}}^{\langle r\rangle}[0], \ldots, \dot{x}_{\mathrm{D} ; \mathrm{I}}^{\langle r\rangle}\left[N_{P}-1\right]\right]^{T}$ and $\dot{\mathbf{X}}_{\mathrm{D} ; \mathrm{I}}^{\langle r\rangle}=\mathbf{F}^{N_{P}} \dot{\mathbf{x}}_{\mathrm{D} ; \mathrm{I}}^{\langle r\rangle}$. According to $(5), h_{1 ; 2}^{\langle r, i\rangle}[n]$ can be estimated with $\dot{x}_{\mathrm{D}}^{\langle r\rangle}[n]$ while $h_{3 ; 4}^{\langle r, i\rangle}[n]$ with $\dot{x}_{\mathrm{I}}^{\langle r\rangle}[n]$. Similar to the FDS estimation scheme in [19], the following relation can be obtained (when ignoring noise):

$$
\dot{\mathbf{X}}_{\mathrm{D} ; \mathrm{I}, \mathscr{F}_{i}}^{\langle r\rangle}=\mathbf{T}^{(i)} \mathbf{F}_{\left(\mathscr{I}_{i}, \mathscr{L}_{\mathrm{h}}\right)}^{N_{P}} \mathbf{h}_{1 ; 3}^{\langle r, i\rangle}, \quad \dot{\mathbf{X}}_{\mathrm{D} ; \mathrm{I},-\mathscr{I}_{i}}^{\langle r\rangle}=\dot{\mathbf{T}}^{(i) *} \mathbf{F}_{\left(-\mathscr{F}_{i}, \mathscr{L}_{\mathrm{h}}\right)}^{N_{P}} \mathbf{h}_{2 ; 4}^{\langle r, i\rangle},
$$

where

$$
\begin{aligned}
& \mathbf{T}^{(i)}:=\operatorname{diag}\left\{\left[T^{(i)}[0], \ldots, T^{(i)}\left[L^{\prime}-1\right]\right]\right\}, \\
& \dot{\mathbf{T}}^{(i)}:=\operatorname{diag}\left\{\left[T^{(i)}\left[L^{\prime}-1\right], \ldots, T^{(i)}[0]\right]\right\}, \\
& \mathbf{h}_{1 ; 2 ; 3 ; 4}^{\langle r, i\rangle}:=\left[h_{1 ; 2 ; 3 ; 4}^{\langle r, i\rangle}[0], \ldots, h_{1 ; 2 ; 3 ; 4}^{\langle r, i\rangle}\left[L_{\mathrm{h}}-1\right]\right]^{T}, \\
& \mathscr{L}_{\mathrm{h}}:=\left\{0, \ldots, L_{\mathrm{h}}-1\right\} .
\end{aligned}
$$

Correspondingly, $\mathbf{h}_{1 ; 2 ; 3 ; 4}^{\langle r, i\rangle}$ can be estimated with the following two methods.

(1) Maximum likelihood estimation (MLE)

$$
\begin{aligned}
& \widehat{\mathbf{h}}_{1 ; 3}^{\langle r, i\rangle}=\left(\mathbf{F}_{\left(\mathscr{I}_{i}, \mathscr{L}_{\mathrm{h}}\right)}^{N_{P}}\right)^{\dagger} \mathbf{T}^{(i) *} \dot{\mathbf{X}}_{\mathrm{D} ; \mathrm{I}, \mathscr{I}_{i}}^{\langle r\rangle}, \\
& \widehat{\mathbf{h}}_{2 ; 4}^{(i)}=\left(\mathbf{F}_{\left(-\mathscr{I}_{i}, \mathscr{L}_{\mathrm{h}}\right)}^{N_{P}}\right)^{\dagger} \dot{\mathbf{T}}^{(i)} \dot{\mathbf{X}}_{\mathrm{D} ; \mathrm{I},-\mathscr{F}_{i}}^{\langle r\rangle} .
\end{aligned}
$$

(2) LMMSE

$$
\widehat{\mathbf{h}}_{1 ; 3}^{\langle r, i\rangle}=\mathfrak{Q}_{\mathrm{D}}^{(i)} \mathbf{T}^{(i) *} \dot{\mathbf{X}}_{\mathrm{D} ; \mathrm{I}, \mathscr{F}_{i}}^{\langle r\rangle}, \quad \widehat{\mathbf{h}}_{2 ; 4}^{(i)}=\mathfrak{Q}_{\mathrm{I}}^{(i)} \dot{\mathbf{T}}^{(i)} \dot{\mathbf{X}}_{\mathrm{D} ; \mathrm{I},-\mathscr{F}_{i}}^{\langle r\rangle},
$$

with

$$
\begin{aligned}
& \mathfrak{Q}_{\mathrm{D}}^{(i)}:=\left(\left(\mathbf{F}_{\left(\mathscr{I}_{i}, \mathscr{L}_{\mathrm{h}}\right)}^{N_{P}}\right)^{H} \mathbf{F}_{\left(\mathscr{I}_{i}, \mathscr{L}_{\mathrm{h}}\right)}^{N_{P}}+\frac{L_{\mathrm{h}}}{\rho^{\langle r\rangle}} \mathbf{I}_{L_{\mathrm{h}}}\right)^{-1}\left(\mathbf{F}_{\left(\mathscr{I}_{i}, \mathscr{L}_{\mathrm{h}}\right)}^{N_{P}}\right)^{H}, \\
& \mathfrak{Q}_{\mathrm{I}}^{(i)}:=\left(\left(\mathbf{F}_{\left(-\mathscr{I}_{i}, \mathscr{L}_{\mathrm{h}}\right)}^{N_{P}}\right)^{H} \mathbf{F}_{\left(-\mathscr{I}_{i}, \mathscr{L}_{\mathrm{h}}\right)}^{N_{P}}+\frac{L_{\mathrm{h}}}{\rho^{\langle r\rangle}} \mathbf{I}_{L_{\mathrm{h}}}\right)^{-1}\left(\mathbf{F}_{\left(-\mathscr{I}_{i}, \mathscr{L}_{\mathrm{h}}\right)}^{N_{P}}\right)^{H},
\end{aligned}
$$

where $\rho^{\langle r\rangle}$ is the SNR at each Rx antenna. To reduce complexity, a fixed value can be assumed for $\rho^{\langle r\rangle}$. In this case, both $\mathfrak{Q}_{\mathrm{D}}^{(i)} \mathbf{T}^{(i) *}$ and $\mathfrak{Q}_{\mathrm{I}}^{(i)} \dot{\mathbf{T}}^{(i)}$ can be regarded as known (i.e., can be precomputed).

4.2.4. Soft Switching Method for Critical CFO Values. In practice, when $\Omega \rightarrow 0$ and $\Omega \rightarrow \pm \pi$, the CLFE in (20) will suffer from high sensitivity to noise [5]. Moreover, the condition number of $\mathfrak{f}_{\Omega}$ will become very large, resulting in large estimation error for $\dot{\mathbf{z}}_{\mathrm{D} ; \mathrm{I}}^{\langle r\rangle}$. Section 6 will show that in such cases, the rough estimator $\widehat{\epsilon}^{\text {rough }}$ will be much more accurate than the CLFE. Thus, the rough estimator should be used. Moreover, instead of applying (23), we should set

$$
\widehat{\dot{\mathbf{z}}}_{\mathrm{D}}^{\langle r\rangle}=\frac{1}{3}\left(\dot{\mathbf{y}}_{1}^{\langle r\rangle}+\dot{\mathbf{y}}_{3}^{\langle r\rangle}+\operatorname{sgn}(\cos \widehat{\Omega}) \dot{\mathbf{y}}_{2}^{\langle r\rangle}\right), \quad \widehat{\dot{\mathbf{z}}}_{\mathrm{I}}^{\langle r\rangle}=\mathbf{0}_{N_{P} \times 1},
$$

which implies that the $\mathrm{Rx}-\mathrm{I} / \mathrm{Q}$-imbalance will not be separately compensated for but jointly with the Tx-I/Q-imbalance and the MIMO channel. For the detection of such cases $(\Omega \rightarrow$ 0 and $\Omega \rightarrow \pm \pi$ ), we observe the relation

$$
\frac{1}{N_{R} N_{P}} E\left\{\left\|\dot{\mathbf{y}}_{13}-2 \operatorname{sgn}(\cos \Omega) \dot{\mathbf{y}}_{2}\right\|_{2}^{2}\right\} \geq 6 \widetilde{\sigma}_{\mathrm{n}}^{2},
$$

which follows from (22) (we assume $E\left\{\left|\widetilde{\eta}^{\langle r\rangle}[n]\right|^{2}\right\}=\widetilde{\sigma}_{n}^{2}$ ). The equality applies when $\Omega=0$ or $\Omega= \pm \pi$. Accordingly, we can define the following metric:

$$
P_{\Delta}:=\frac{1}{N_{R} N_{P}}\left\|\dot{\mathbf{y}}_{13}-2 \operatorname{sgn}(\cos \Omega) \dot{\mathbf{y}}_{2}\right\|_{2}^{2}-6 \zeta \widetilde{\sigma}_{\mathrm{n}}^{2},
$$

where $\zeta \geq 1$ is an adjusting factor. Once $P_{\Delta}<0$, both $\widehat{\epsilon}^{\text {rough }}$ and (31) are applied. Otherwise, we apply $\left\|\mathfrak{f}_{\widehat{\Omega}} \widehat{\dot{\vec{j}}}\right\|_{F}^{2}$, which corresponds to the power of the signal part in (22), as a further metric to switch between $\widehat{\epsilon}$ and $\widehat{\epsilon}^{\text {rough }}$, where $\widehat{\dot{3}}:=\left[\widehat{\dot{3}}^{\langle 1\rangle}, \ldots, \widehat{\dot{3}}^{\left\langle N_{R}\right\rangle}\right]$. The estimator that leads to a larger $\| \mathfrak{f}_{\widehat{\Omega}} \widehat{\dot{S}}_{F}^{2}$ is chosen. Compared to [7], the metrics above allow soft switching.

4.2.5. Iterative Improvement. To further improve the CFO estimation and the separation of $\dot{z}_{\mathrm{D}}^{\langle r\rangle}[n]$ and $\dot{z}_{\mathrm{I}}^{\langle r\rangle}[n]$, the following iterative processing can be applied:

(1) $\dot{\mathbf{y}}_{b}^{\langle r\rangle}=\dot{\mathbf{y}}_{b}^{\langle r\rangle}-\hat{\mathbf{z}}_{\mathrm{I}}^{\langle r\rangle} e^{-j \widehat{\Omega}(b-1)}$, for all $b, r$ is calculated to eliminate the influence of $\mathrm{Rx}-\mathrm{I} / \mathrm{Q}$-imbalance on the received preamble, 
(2) equation (21) is applied with $\dot{\mathbf{y}}_{b}$ instead of $\dot{\mathbf{y}}_{b}$ to obtain a new estimate of $\epsilon$,

(3) with the new estimate of $\epsilon,(23)$ is used to obtain a new version of $\widehat{\dot{3}}^{\langle r\rangle}$, for all $r$.

The above calculation can be carried out iteratively until a predefined allowable iteration number is exceeded.

4.3. Calculation of Compensation Coefficients. Now, we have the estimates of $\epsilon$ and $h_{1}^{\langle r, i\rangle}[n] \sim h_{4}^{\langle r, i\rangle}[n]$. According to Section 3, successful Rx-I/Q-imbalance compensation requires the fulfillment of the two conditions in (12). Since both conditions are equivalent, we only have to choose one of them. We consider that with realistic I/Q-imbalance parameters, $h_{\mathrm{D}, \mathrm{T} ; \mathrm{R}}^{(i) ; r\rangle}[n]$ would have much larger power gain than $h_{\mathrm{I}, \mathrm{T} ; \mathrm{R}}^{(i) ;\langle n\rangle}[n]$. Thus, according to $(6), h_{1}^{\langle r, i\rangle}[n]$ would have much larger power gain than $h_{2 ; 3 ; 4}^{\langle r, i\rangle}[n]$ and can be estimated with much lower estimation error (caused by noise). According to (10), $\vartheta_{\mathrm{I}}^{E\langle r, i\rangle}[n]$ consists partly of $h_{1}^{\langle r, i\rangle}[n]$. Thus, it should be used to obtain reliable compensation coefficients. From (4), (9), and (12), we obtain

$$
\left(1+j c^{\langle r\rangle}\right) \gamma_{1}^{\langle r, i\rangle}[n]+h^{C\langle r\rangle}[n] * \gamma_{2}^{\langle r, i\rangle}[n]=0, \quad \forall i,
$$

with

$$
\begin{aligned}
& \gamma_{1}^{\langle r, i\rangle}[n]:=\left(h_{1}^{\langle r, i\rangle *}\left[n-n_{\tau}\right]+h_{4}^{\langle r, i\rangle}\left[n-n_{\tau}\right]\right) e^{-j(2 \pi / N) \epsilon\left(n-n_{\tau}\right)} \\
& \gamma_{2}^{\langle r, i\rangle}[n]:=\left(-h_{1}^{\langle r, i\rangle *}[n]+h_{4}^{\langle r, i\rangle}[n]\right) e^{-j(2 \pi \epsilon n / N)} .
\end{aligned}
$$

Equation (34) can be rewritten with real-valued signals as

$$
\begin{aligned}
& -\gamma_{1, \mathrm{re}}^{\langle r, i\rangle}[n]=-c \gamma_{1, \mathrm{im}}^{\langle r, i\rangle}[n]+h^{C\langle r\rangle}[n] * \gamma_{2, \mathrm{re}}^{\langle r, i\rangle}[n], \quad \forall i, \\
& -\gamma_{1, \mathrm{im}}^{\langle r, i\rangle}[n]=c \gamma_{1, \mathrm{re}}^{\langle r, i\rangle}[n]+h^{C\langle r\rangle}[n] * \gamma_{2, \mathrm{im}}^{\langle r, i\rangle}[n], \quad \forall i .
\end{aligned}
$$

Let $V_{1 ; 2}^{\mathrm{Re} ; \operatorname{Im},\langle r, i\rangle}[k]:=\mathscr{F}_{N_{P}}\left\{\gamma_{1 ; 2, \mathrm{re} ; \mathrm{im}}^{\langle r, i\rangle}[n]\right\}$ and $H^{C\langle r\rangle}[k]:=$ $\mathscr{F}_{N_{P}}\left\{h^{C\langle r\rangle}[n]\right\}$. Equation (36) yields

$$
\begin{aligned}
& \underbrace{\left[\begin{array}{c}
-V_{1}^{\operatorname{Re}\langle r, 1\rangle}[k] \\
-V_{1}^{\operatorname{Im}\langle r, 1\rangle}[k] \\
\vdots \\
-V_{1}^{\operatorname{Re}\left\langle r, N_{T}\right\rangle}[k] \\
-V_{1}^{\operatorname{Im}\left\langle r, N_{T}\right\rangle}[k]
\end{array}\right]}_{\boldsymbol{v}_{k}^{\langle r\rangle}} \\
& =\underbrace{\left[\begin{array}{cc}
-V_{1}^{\operatorname{Im}\langle r, 1\rangle}[k] & V_{2}^{\operatorname{Re}\langle r, 1\rangle}[k] \\
V_{1}^{\operatorname{Re}\langle r, 1\rangle}[k] & V_{2}^{\operatorname{Im}\langle r, 1\rangle}[k] \\
\vdots & \vdots \\
-V_{1}^{\operatorname{Im}\left\langle r, N_{T}\right\rangle}[k] & V_{2}^{\operatorname{Re}\left\langle r, N_{T}\right\rangle}[k] \\
V_{1}^{\operatorname{Re}\left\langle r, N_{T}\right\rangle}[k] & V_{2}^{\operatorname{Im}\left\langle r, N_{T}\right\rangle}[k]
\end{array}\right]}_{\Theta_{k}^{\langle r\rangle}} \underbrace{\left[\begin{array}{c}
c^{\langle r\rangle} \\
H^{C\langle r\rangle}[k]
\end{array}\right]}_{\lambda_{k}^{\langle r\rangle}} .
\end{aligned}
$$

Thus, $\boldsymbol{\lambda}_{k}^{\langle r\rangle}$ can be estimated by

$$
\hat{\boldsymbol{\lambda}}_{k}^{\langle r\rangle}=\left(\boldsymbol{\Theta}_{k}^{\langle r\rangle}\right)^{\dagger} \boldsymbol{v}_{k}^{\langle r\rangle}
$$

Afterwards, $c^{\langle r\rangle}$ and $h^{C\langle r\rangle}[n]$ can be calculated by

$$
\widehat{c}^{\langle r\rangle}=\frac{1}{N_{D, P}} \sum_{k \in \mathscr{F}_{D, P}}\left(\hat{\lambda}_{k}^{\langle r\rangle}\right)_{1}, \quad \widehat{\mathbf{h}}^{C\langle r\rangle}=\left(\mathbf{F}_{\left(\mathscr{I}_{D, P}, \mathscr{L}\right)}\right)^{\dagger} \widehat{\mathbf{H}}_{\mathscr{I}_{D, P}}^{C},
$$

with $\mathscr{I}_{D, P}:=\left\{-N_{D, P} / 2, \ldots,-1,1, \ldots, N_{D, P} / 2\right\}, \mathbf{h}^{C\langle r\rangle}:=$ $\left[h^{C\langle r\rangle}[0], \ldots, h^{C\langle r\rangle}\left[L_{c}-1\right]\right]^{T}, \mathscr{L}:=\left\{0, \ldots, L_{c}-1\right\}$, and $\mathbf{H}^{C\langle r\rangle}$ is a length $N_{P}$ column vector with $\left(\mathbf{H}^{C\langle r\rangle}\right)_{k}=H^{C\langle r\rangle}[k]$. Note that Tx antenna diversity is exploited in this calculation. Moreover, the influence of guardband is taken into account in (39). Finally, since $V_{1 ; 2}^{\mathrm{Re} ; \operatorname{Im}\langle r, i\rangle}[k]$ is a conjugate symmetrical function of $k$, the calculation of (38) only needs to be carried out for $1 \leq k \leq N_{D, P} / 2$.

To further reduce computational complexity, the DFT block size of $V_{1 ; 2}^{\mathrm{Re}, \operatorname{Im},\langle r, i\rangle}[k]$ can be reduced to a number $N_{\mathrm{V}}$ which just has to be larger than $L_{\mathrm{h}}+L_{\mathrm{c}}+n_{\tau}$. Furthermore, instead of $\mathscr{I}_{D, P}$, a proper subcarrier index set $\mathscr{I}_{D, V}$, with $\left|\mathscr{I}_{D, V}\right|=N_{D, V}$, should be defined according to the guardband size and the sampling rate.

Finally, with (10) and (9), we can calculate $h_{\mathrm{D} ; \mathrm{I}}^{E\langle r, i\rangle}[n]$, that is, also $H_{\mathrm{D} ; \mathrm{I}}^{E\langle r, i\rangle}[k]$.

\section{Joint Estimation Scheme 2: Iterative CFO and Rx-I/Q-Imbalance Estimation Method}

This scheme is developed directly based on the compensation structure in Figure 5. This scheme uses a similar preamble. First, the preamble is applied to estimate $\epsilon, c^{\langle r\rangle}$, and $h^{C,\langle r\rangle}[n]$ in an iterative manner. Afterwards, the influence of the CFO and Rx-I/Q-imbalance on the preamble is eliminated. Finally, the coefficients $H_{\mathrm{D} ; \mathrm{I}}^{E\langle r, i\rangle}[k]$ are calculated.

Note that the basic idea of the CFO and Rx-I/Qimbalance estimation is similar to that in [15]. However, our scheme is developed based on an Rx-I/Q-imbalance compensation structure with real-valued coefficients, which allows much lower computational complexity (both for parameter estimation and the actual compensation). Moreover, practical extension is developed to cope with the troublesome cases of critical CFO values, that is, $\Omega \rightarrow 0$ and $\Omega \rightarrow \pm \pi$.

5.1. Preamble Design. This scheme applies a similar preamble design as in Section 4.1. The same basic sequence is applied for each Tx antenna. However, the repetition number of this basic sequence can be as low as two. Let $N_{\text {rep }}$ be the number of repetitions, then the total length of the preamble is $N_{\mathrm{CP}}+$ $N_{\text {rep }} * N_{P}$. 


\subsection{Estimation Scheme}

5.2.1. Iterative $C F O$ and $R x-I / Q-I m b a l a n c e$ Estimation. For simplicity of description, we first assume that $N_{\text {rep }}=2$. The extension to preambles with $N_{\text {rep }}>2$ will be shown later. Similar to Section 4.2, $\dot{y}_{b}^{\langle r\rangle}[n]$ indicates the received signal of the $b$ th repetition of the basic sequence. This estimation scheme is based on the observation that, assuming perfect CFO knowledge, perfect $\mathrm{Rx}-\mathrm{I} / \mathrm{Q}$-imbalance compensation coefficients, and no noise, we have for each $\mathrm{Rx}$ - antenna

$$
\left(\dot{\mathbf{y}}_{\mathrm{re}, 1}^{\langle r\rangle}+j \dot{\mathbf{Y}}_{\mathrm{CB}, 1}^{\langle r\rangle} \mathbf{h}^{\mathrm{C}, \mathrm{Ext},\langle r\rangle}\right) e^{j \Omega}=\dot{\mathbf{y}}_{\mathrm{re}, 2}^{\langle r\rangle}+j \dot{\mathbf{Y}}_{\mathrm{CB}, 2}^{\langle r\rangle} \mathbf{h}^{\mathrm{C}, \mathrm{Ext},\langle r\rangle},
$$

with

$$
\dot{\mathbf{Y}}_{\mathrm{CB}, b}^{\langle r\rangle}:=\left[\dot{\mathbf{y}}_{\mathrm{re}, b}^{\langle r\rangle}, \dot{\mathbf{Y}}_{\mathrm{im}, b}^{\langle r\rangle}\right], \quad \mathbf{h}^{\mathrm{C}, \mathrm{Ext},\langle r\rangle}:=\left[c^{\langle r\rangle},\left(\mathbf{h}^{\mathrm{C},\langle r\rangle}\right)^{T}\right]^{T},
$$

where $\dot{\mathbf{Y}}_{\mathrm{im}, b}^{\langle r\rangle}$ is an $N_{P} \times L_{\mathrm{c}}$ Toeplitz matrix with the $n_{\tau}^{\text {th }}$ column equal to $\dot{\mathbf{y}}_{\mathrm{im}, b}^{\langle r\rangle}$.

Equation (40) can be rewritten as

$$
\underbrace{\left[\begin{array}{c}
\dot{\mathbf{y}}_{\mathrm{re}, 1}^{\langle r\rangle} \cos \Omega-\dot{\mathbf{y}}_{\mathrm{re}, 2}^{\langle r\rangle} \\
\dot{\mathbf{y}}_{\mathrm{re}, 1}^{\langle r\rangle} \sin \Omega
\end{array}\right]}_{\mathbf{T}_{e}^{\langle r\rangle}}=\underbrace{\left[\begin{array}{c}
\dot{\mathbf{Y}}_{\mathrm{CB}, 1}^{\langle r\rangle} \sin \Omega \\
\dot{\mathbf{Y}}_{\mathrm{CB}, 2}^{\langle r\rangle}-\dot{\mathbf{Y}}_{\mathrm{CB}, 1}^{\langle r\rangle} \cos \Omega
\end{array}\right]}_{\mathbf{R}_{e}^{\langle r\rangle}} \mathbf{h}^{\mathrm{C}, \mathrm{Ext},\langle r\rangle} .
$$
by

According to (42), if $\epsilon$ is known, $\mathbf{h}^{\mathrm{C}, \mathrm{Ext},\langle r\rangle}$ can be estimated

$$
\widehat{\mathbf{h}}^{\mathrm{C}, \mathrm{Ext},\langle r\rangle}=\mathbf{R}_{\epsilon}^{\langle r\rangle^{\dagger}} \mathbf{T}_{\epsilon}^{\langle r\rangle}
$$

Thus, we first carry out an initial CFO estimation by

$$
\widehat{\boldsymbol{\epsilon}}^{\text {init }}=\frac{N}{2 \pi N_{P}} \arg \left\{\left(\dot{\mathbf{y}}_{1}^{T}\right)^{*} \dot{\mathbf{y}}_{2}\right\},
$$

where $\dot{\mathbf{y}}_{b}$, for all $b=1,2$, is defined as in Section 4.2. Afterwards, the initial CFO estimate is applied to (43) to obtain an initial estimate of $\mathbf{h}^{\mathrm{C}, \mathrm{Ext},\langle r\rangle}$, for all $r$, which is used to carry out $\mathrm{Rx}-\mathrm{I} / \mathrm{Q}$-imbalance compensation on the received preambles as follows:

$$
\dot{\mathbf{y}}_{b}^{\langle r\rangle}=\dot{\mathbf{y}}_{\mathrm{re}, b}^{\langle r\rangle}+j \dot{\mathbf{Y}}_{\mathrm{CB}, b}^{\langle r\rangle} \mathbf{h}^{\mathrm{C}, \mathrm{Ext},\langle r\rangle}
$$

Now, the operation in (44) is applied again, but with $\dot{\mathbf{y}}_{b}^{\langle r\rangle}$ instead of $\dot{\mathbf{y}}_{b}^{\langle r\rangle}$, to obtain a new estimate of $\epsilon$. This new CFO estimate is applied again to (43) to obtain a new estimate of $\mathbf{h}^{\mathrm{C}, \mathrm{Ext},\langle r\rangle}$, for all $r$. Afterwards, Rx-I/Q-imbalance compensation is carried out with this new estimate. This process is repeated iteratively until a predefined allowable iteration number is exceeded.

5.2.2. Estimation of $h_{\mathrm{D}}^{E\langle r, i\rangle}[n]$ and $h_{\mathrm{I}}^{E\langle r, i\rangle}[n]$. After all iterations, a final CFO and Rx-I/Q-imbalance compensation is carried out on the received preamble sequences according to
Figure 5. We denote the received preamble sequences after this compensation as $\check{\mathbf{y}}_{b}^{\langle r\rangle}$. First, we carry out averaging over the two repetitions to mitigate noise influence

$$
\overline{\mathbf{y}}^{\langle r\rangle}=\frac{1}{2} \sum_{b=1}^{2} \check{\mathbf{y}}_{b}^{\langle r\rangle} .
$$

Let $\overline{\dot{\mathbf{Y}}}^{\langle r\rangle}:=\mathbf{F}^{N_{P}} \overline{\dot{\mathbf{y}}}^{\langle r\rangle}$. If we ignore the noise influence, the following relation exists:

$$
\overline{\dot{\mathbf{Y}}}_{\mathscr{F}_{i}}^{\langle r\rangle}=\mathbf{T}^{(i)} \mathbf{F}_{\left(\mathscr{I}_{i}, \mathscr{L}_{\mathrm{h}}\right)}^{N_{P}} \mathbf{h}_{\mathrm{D}}^{E\langle r, i\rangle}, \quad \overline{\dot{\mathbf{Y}}}_{-\mathscr{I}_{i}}^{\langle r\rangle}=\dot{\mathbf{T}}^{(i) *} \mathbf{F}_{\left(-\mathscr{I}_{i}, \mathscr{L}_{\mathrm{h}}\right)}^{N_{P}} \mathbf{h}_{\mathrm{I}}^{E\langle r, i\rangle} .
$$

Finally, we can obtain $\widehat{\mathbf{h}}_{\mathrm{D}}^{E\langle r, i\rangle}$ and $\widehat{\mathbf{h}}_{\mathrm{I}}^{E\langle r, i\rangle}$ either using MLE

$$
\begin{aligned}
& \widehat{\mathbf{h}}_{\mathrm{D}}^{E\langle r, i\rangle}=\left(\mathbf{F}_{\left(\mathscr{F}_{i}, \mathscr{L}_{\mathrm{h}}\right)}^{N_{P}}\right)^{\dagger} \mathbf{T}^{(i) *} \overline{\dot{\mathbf{Y}}}_{\mathscr{I}_{i}}^{\langle r\rangle}, \\
& \widehat{\mathbf{h}}_{\mathrm{I}}^{E\langle r, i\rangle}=\left(\mathbf{F}_{\left(-\mathscr{F}_{i}, \mathscr{L}_{\mathrm{h}}\right)}^{N_{P}}\right)^{\dagger} \mathbf{T}^{(i)} \overline{\mathbf{Y}}_{-\mathscr{I}_{i}}^{\langle r\rangle},
\end{aligned}
$$

or using LMMSE

$$
\widehat{\mathbf{h}}_{\mathrm{D}}^{E\langle r, i\rangle}=\mathfrak{Q}_{\mathrm{D}}^{(i)} \mathbf{T}^{(i) *} \overline{\dot{\mathbf{Y}}}_{\mathscr{I}_{i}}^{\langle r\rangle}, \quad \widehat{\mathbf{h}}_{\mathrm{I}}^{E\langle r, i\rangle}=\mathfrak{Q}_{\mathrm{I}}^{(i)} \dot{\mathbf{T}}^{(i)} \overline{\dot{\mathbf{Y}}}_{-\mathscr{I}_{i}}^{\langle r\rangle},
$$

where $\mathfrak{Q}_{D ; I}$ is defined as in (30). Finally, we calculate

$$
\widehat{H}_{\mathrm{D} ; \mathrm{I}}^{E\langle r, i\rangle}[k]=\mathscr{F}_{N}\left\{\widehat{h}_{\mathrm{D} ; \mathrm{I}}^{E\langle r, i\rangle}[n]\right\} \text {. }
$$

5.2.3. Soft Switching Method for Critical CFO Values. Similar to the scheme in Section 4.2, the estimation scheme previous will have poor performance when $\Omega \rightarrow 0$ or $\Omega \rightarrow \pm \pi$. The reason is that this scheme utilizes the negative phase rotation caused by $\mathrm{CFO}$ to identify the Rx-I/Q-imbalance characteristic. However, this identification is impossible when $\Omega=0$ or $\Omega= \pm \pi$. Actually, if we ignore the noise in these cases, $\mathbf{R}_{\epsilon}^{\langle r\rangle}$ and $\mathbf{T}_{\epsilon}^{\langle r\rangle}$ in (43) will become zero valued.

To avoid this problem, we observe the following relation:

$$
\frac{1}{N_{P}} E\left\{\left\|\dot{\mathbf{y}}_{1} e^{j \Omega}-\dot{\mathbf{y}}_{2}\right\|_{2}^{2}\right\} \geq 2 \widetilde{\sigma}_{\mathrm{n}}^{2},
$$

from which the following soft-metric can be defined:

$$
P_{\Delta}^{\prime}:=\frac{1}{N_{P}}\left\|\dot{\mathbf{y}}_{1} e^{j\left(2 \pi \hat{\epsilon} N_{P} / N\right)}-\dot{\mathbf{y}}_{2}\right\|_{2}^{2}-2 \zeta \widetilde{\sigma}_{\mathrm{n}}^{2},
$$

with $\zeta$ as an adjusting factor. If $P_{\Delta}^{\prime}>0$, the iterative estimation scheme can be applied. However, if $P_{\Delta}^{\prime} \leq 0$, we can assume that $\Omega \rightarrow 0$ or $\Omega \rightarrow \pm \pi$. In this case, we should omit the iterative estimation. Moreover, the initial CFO estimation is used to carry out CFO correction on the received preamble as in the case without $\mathrm{Rx}$-I/Q-imbalance. Based on the corrected preamble, joint Tx- and Rx-I/Qimbalance and MIMO channel estimation is carried out with the FDS-preamble-based scheme in [19]. This implies that no separate Rx-I/Q-imbalance compensation is applied. 
The proposed estimation scheme can be easily applied to preambles containing more than two repetitions of the basic sequence. To enable this, we just need to reorder the multiple basic repetitions into two augmented repetitions. These two augmented repetitions are allowed to have overlapped areas.

\section{Simulation Results}

6.1. Simulation Setups. In the simulation, the amplitude and phase imbalance of the modulator/demodulator are about $5 \%$ and $5^{\circ}$, respectively. The LPFs in the I and Q branches (in all $\mathrm{Tx} / \mathrm{Rx}$ branches) have relative amplitude mismatch and phase differences of up to $10 \%$ and $10^{\circ}$, respectively. All different $\mathrm{Tx}$ and $\mathrm{Rx}$ branches have different I/Q-imbalance parameters. All imbalance parameters are assumed to be time invariant. Furthermore, the measured $60 \mathrm{GHz}$ MIMO channels in [20] were used. The following OFDM parameter sets are investigated: $N=256, N_{0}=17$, and $N=512, N_{0}=$ 91. Furthermore, we apply $N_{T}=2, N_{R}=2, L_{\mathrm{h}}=32$, and $L_{\mathrm{c}}=8$. Both the closed-form-based scheme in Section 4 (indicated as "SCH1") and the iterative scheme in Section 5 (indicated as "SCH2") were applied to estimate CFO, Tx- and $\mathrm{Rx}-\mathrm{I} / \mathrm{Q}$-imbalance, and the MIMO channel. For "SCH1", the LSE (applying (24)) and the LMMSE estimation (applying (25)) of $\dot{3}^{\langle r\rangle}$ are compared. (Since the estimation of $\dot{3}^{\langle r\rangle}$ corresponds to the separation of $\dot{\mathbf{z}}_{\mathrm{D}}^{\langle r\rangle}$ and $\dot{\mathbf{z}}_{\mathrm{I}}^{\langle r\rangle}$, it is indicated by "SEP" in the simulation results.) For "SCH2", the application of the real-valued Rx-I/Q-imbalance compensation structure in Figure 5 is compared with that of a complex-valued $\mathrm{Rx}^{-}$ I/Q-imbalance compensation structure, which is described in the appendix (or in [15]). For "SCH1" and "SCH2", both MLE and LMMSE (both MLE and LMMSE can be decomposed in to two steps: first, LSE of coefficients on pilot subcarriers; second, Interpolation of the LSE. Since the difference between MLE and LMMSE only lies in the interpolation, the corresponding simulation results are indicated by "INTP") of $H_{\mathrm{D} ; \mathrm{I}}^{E}$ (corresponding to (28), (48), (29), and (49), resp.) are compared. When applying LMMSE, we assume a fixed value $10 \log _{10} \rho^{\langle r\rangle}=30 \mathrm{~dB}$, for all $r$; for the calculation of $\mathfrak{Q}_{\mathrm{D} ; \mathrm{I}}$ in (30). For a fair comparison between "SCH1" and "SCH2," the same preamble was applied. No matter $N=256$ or $N=512$, the applied preamble consists of 3 repetitions of a basic sequence with $N_{P}=256$, (Note that the preamble length is not directly related to the OFDM symbol length but the parameters $N_{T}$ and $L_{\mathrm{h}}$ (see Section 4.1).) Thus, the total preamble length was 800 . To apply "SCH2," the three repetitions were reordered to two augmented repetitions of length 512(with an overlapping area of 256 samples) as described in Section 5.2.

As reference, the original iterative estimation scheme of [15] was also applied, which is indicated as "Hsu." This scheme uses a nonoptimal preamble, which consists of two parts. The first part consists of $M_{\mathrm{ST}}$ repetitions of a length $N_{\mathrm{ST}}$ short training sequence and is used for $\mathrm{CFO}$ and $\mathrm{Rx}-\mathrm{I} / \mathrm{Q}$-imbalance estimation. The second half consists of $M_{\mathrm{LT}}$ repetitions of a length $N_{\mathrm{LT}}$ long training sequence (each is attached to a CP) and is used for the estimation of $H_{\mathrm{D} ; \mathrm{I}}^{E}[k]$, for all $k$ (these long training sequences are constructed as OFDM symbols whose subcarriers have constant amplitude and random phases). Thus, the total preamble length is $N_{\mathrm{TL}}=M_{\mathrm{ST}} N_{\mathrm{ST}}+M_{\mathrm{LT}} N_{\mathrm{LT}}+$ $\left(M_{\mathrm{LT}}+1\right) N_{\mathrm{CP}}$. Two cases of the "Hsu" scheme were observed. The first case (indicated as "Hsu S") is that "Hsu" has the same preamble length as "SCH1/SCH2," that is, $N_{\mathrm{TL}}=800$. Correspondingly, $M_{\mathrm{ST}}=5, N_{\mathrm{ST}}=64, M_{\mathrm{LT}}=2$, and $N_{\mathrm{LT}}=192$. The second case (indicated as "Hsu L") is that the first part of the preamble is already of length 800 , with $M_{\mathrm{ST}}=$ 12 and $N_{\mathrm{ST}}=64$. The second part of the preamble contains $M_{\mathrm{LT}}=2$ OFDM symbols of regular length $\left(N_{\mathrm{LT}}=N\right)$. We will show that even with such a long preamble, "Hsu L" is still outperformed by our proposed schemes. Note that the CFO and $\mathrm{Rx}-\mathrm{I} / \mathrm{Q}$-imbalance estimation scheme of "Hsu" is almost the same as that of "SCH2, C" (the Appendix), except for the soft switching. To apply the "Hsu" scheme, the short training sequences are reordered into two augmented repetitions. The first and the second augmented repetitions contain the first and the last $\left(M_{\mathrm{ST}}-1\right)$ th short training sequences, respectively.

Table 1 gives an overview of the abbreviation used in the simulation results. Table 2 lists the preamble lengths of the different schemes.

6.2. Estimation Mean Square Error (MSE) as a Function of the CFO Value. Figure 6 shows the estimation MSEs (unnormalized MSE is used for the estimation of both CFO and Rx-I/Qimbalance compensation coefficients, while normalized MSE is used for the estimation of $H_{\mathrm{D} ; \mathrm{I}}^{E}$ ) of the CFO, the Rx-I/Qimbalance compensation coefficients, and $H_{\mathrm{D} ; \mathrm{I}}^{E}$ as functions of $\epsilon$, with $N=256$ and SNR $=20$. (Since the MSE behavior with $\epsilon<0$ is generally symmetric to that with $\epsilon>0$, we only show the case with $\epsilon>0$. Furthermore, the results with $N=512$ are similar. When $N=512$, the observed $\epsilon$ range becomes $0 \sim 1$.) For comparison, the CFO estimations of (20), indicated by "cosine", and that of (21), indicated by "rough," are included. As shown, the CFO estimat MSEs of both the "cosine" and the "rough" estimators depend strongly on $\epsilon$. From Figure 6, we can see that the "rough" estimator outperforms the "cosine" estimator for a large range of $\epsilon$ values. Furthermore, the soft switching method and iterative improvement proposed in "SCH1" allow MSE that is close to the lower one between "cosine" and "rough". "SCH1" with "SEP2" especially, can achieve much lower MSE than both "cosine" and "rough." Compared to "SCH1", both "SCH2" and Hsu's schemes have CFO estimation MSE that is less dependent on $\epsilon$, where "Hsu, S" has relatively high MSE floor. With most of the $\epsilon$ values, "SCH2, R/C" leads to the lowest CFO estimation MSE.

With all the proposed schemes, the MSEs of the RxI/Q-imbalance compensation coefficients and $H_{\mathrm{D} ; \mathrm{I}}^{E}$ show " $U$ " shapes over the observed $\epsilon$ range. The highest MSEs are found with $\epsilon$ values close to 0 or 0.5 . The reason was that with such values, the distance between $e^{j \Omega}$ and $e^{-j \Omega}$ becomes quite small, leading to difficulties in the identification of the mirror interference generated by Rx-I/Q-imbalance. Although Hsu's schemes can achieve similar Rx-I/Q-imbalance estimation MSE as the proposed schemes, they have much poorer estimations of $H_{\mathrm{D} ; \mathrm{I}}^{E}$ due to nonoptimized preamble design (nonoptimized in avoidance of inter-Tx-antenna interference and 
TABLE 1: Abbreviations in simulation results.

\begin{tabular}{ll}
\hline Abbreviation & Meaning \\
\hline SCH1 & The closed form based joint estimation scheme in Section 4 \\
SCH2 & The iterative joint estimation scheme in Section 5 \\
SEP1 & For SCH1: estimation of $\dot{3}^{\langle r\rangle}$ using LSE, that is, applying (24) \\
SEP2 & For SCH1: estimation of $\dot{3}^{\langle r\rangle}$ using LMMSE, that is, applying (25) \\
R & For SCH2: apply the real-valued Rx-I/Q-imbalance compensation structure (Section 5.2) \\
C & For SCH2: apply the complexed valued Rx-I/Q-imbalance compensation structure as in The appendix \\
INTP1 & For SCH1/SCH2: using the MLE in (28) or (48), respectively \\
INTP2 & For SCH1/SCH2: using the LMMSE in (29) or (49), respectively \\
Hsu, S & The scheme in [15], with a preamble of length 800 \\
Hsu, L & The scheme in [15], with a much longer preamble \\
\hline
\end{tabular}

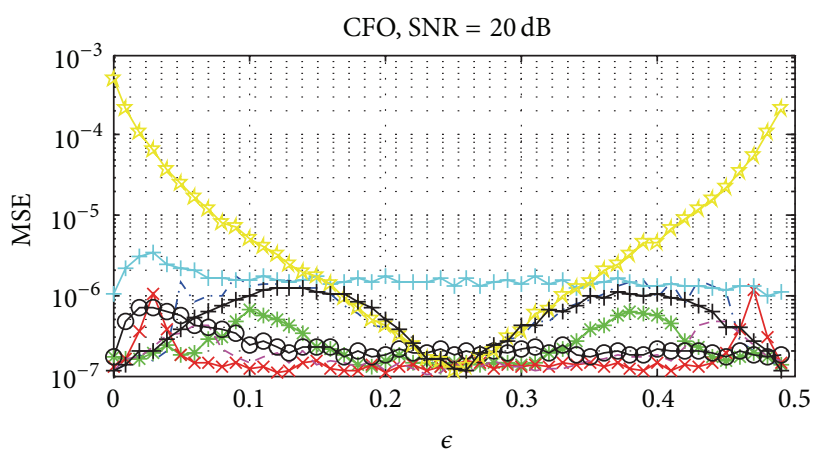

$\begin{array}{ll}-- \text { SCH1, SEP1, INTP2 } & - \text { Hsu, S } \\ * \text { - SCH1, SEP2, INTP2 } & \rightarrow \text { Hsu, L } \\ \text { * SCH2, R, INTP2 } & \text { * Cosine } \\ --- \text { SCH2, C, INTP2 } & \rightarrow \text { Rough }\end{array}$

(a)

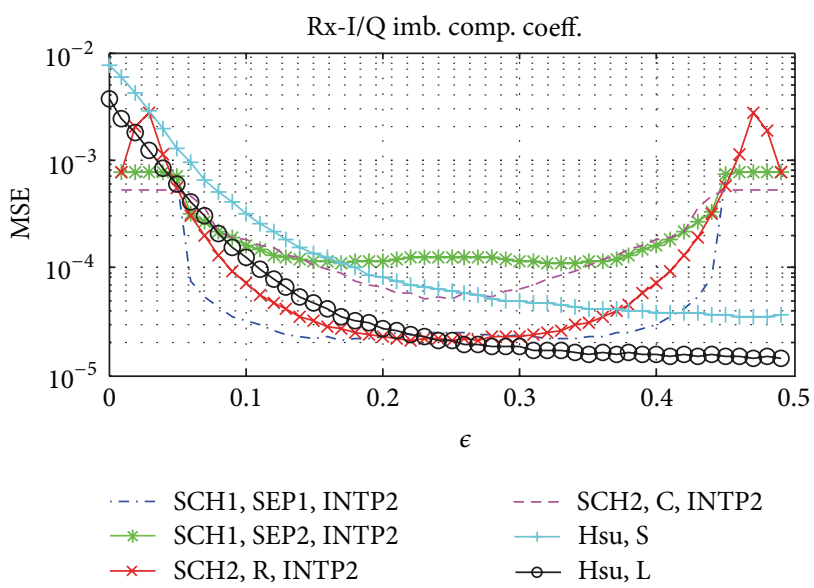

(b)

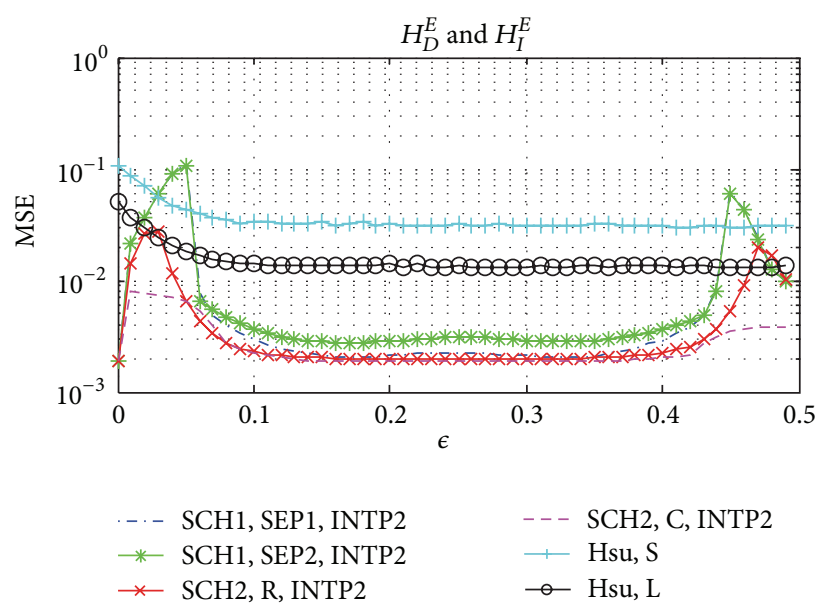

(c)

FIgURE 6: Estimation MSE as a function of $\epsilon$ with $N_{I}=1, \quad N=256$, and SNR $=20 \mathrm{~dB}$.

mirror interference). Note that the "Hsu, S/L" schemes do not have MSE increase for $\epsilon$ values close to 0.5 , since a smaller repetition distance was applied $\left(N_{\mathrm{ST}}=64\right)$, which allows a larger range of $\mathrm{CFO}$ estimation range.

By comparing the MSE results of $\mathrm{CFO}$ and Rx-I/Qimbalance compensation coefficients, we can see that for "SCH1", "SEP1" allows better estimation of Rx-I/Q-imbalance compensation coefficients, while "SEP2" can lead to better CFO estimation (better CFO estimation of "SEP2" is mainly achieved in the case of relatively low SNR). Thus, we suggest to apply "SEP2" within the iterations to obtain CFO estimation and to apply "SEP1" in the final iteration to obtain the estimation of Rx-I/Q-imbalance compensation coefficients. 
TABLE 2: Preamble lengths of different schemes (in number of samples).

\begin{tabular}{lcc}
\hline Schemes & $N=256$ & $N=512$ \\
\hline SCH1; SCH2; Hsu, S & 800 & 800 \\
Hsu, L & 1376 & 1888 \\
\hline
\end{tabular}

6.3. Estimation MSE as a Function of SNR. Figures 7 and 8 show the estimation MSE of all relevant quantities as functions of SNR with $\left\{N=256, N_{0}=17, \epsilon=0.25\right\}$ and $\left\{N=512, N_{0}=91, \epsilon=0.5\right\}$, respectively. As reference, the Cramer-Rao lower bounds (CRLBs) are included. As shown, the proposed schemes can achieve MSE close to the CRLB. It is also shown that when the guardband is small, the performance with LMMSE ("INTP2") is similar to that with MLE ("INTP1"). However, when the guardband is large, the performance with LMMSE ("INTP2") is significantly better. (For "SCH1", the choice between MLE and LMMSE affects both the estimation of the $\mathrm{Rx}-\mathrm{I} / \mathrm{Q}$-imbalance compensation coefficients and $H_{\mathrm{D} ; \mathrm{I}}^{E}$. However, for "SCH2," this choice only has influence on the estimation of $H_{\mathrm{D} ; \mathrm{I}}^{E}$.) As mentioned in Sections 4.2 and 5.2, with a fixed assumed $\rho$ value, the computational complexity of the LMMSE is identical to that of the MLE. Thus, we suggest to apply LMMSE. Furthermore, the CFO and Rx-I/Q-imbalance estimation MSE of the proposed schemes are similar to that of "Hsu, L" scheme, while the MSE of $H_{\mathrm{D} ; \mathrm{I}}^{E}$ with the proposed schemes (applying LMMSE) is much lower than that with Hsu's scheme. Remember that the "Hsu, L" scheme requires much higher preamble overhead than the proposed schemes (see Table 2).

6.4. Estimation MSE as a Function of the Iteration Number. Figure 9 shows the MSE of all related quantities as a function of the iteration number $N_{I}$ with $\{N=256, \epsilon=0.15, \mathrm{SNR}=$ $20 \mathrm{~dB}\}$. The results for other $\epsilon$ - and SNR values as well as with $N=512$ were found to be similar. (For "SCH1", the iteration gain of CFO estimation decreases as $\epsilon$ approaches 0.25 (for $N=256$ ), since both "cosine"- and "rough" estimators can already provide very good estimation accuracy.) As shown, all the schemes have MSE improvement of CFO estimation at the first iteration, where the improvement of "SCH2" is larger than that of "SCH1." We can also observe that further iteration only leads to negligible improvement or even slight degradation. In contrast, the MSE of the Rx-I/Q-imbalance compensation coefficients and $H_{\mathrm{D} ; \mathrm{I}}^{E}$ with all schemes is quite independent of $N_{I}$. Based on these results, we suggest to apply $N_{I}=1$ for all the schemes to achieve a tradeoff between performance and complexity.

6.5. BER as a Function of SNR and the CFO Value. Aside from the cases with the proposed schemes and Hsu's schemes, the following cases are also included in the BER simulation as reference: (1) with a CFO and channel estimation and compensation scheme ignoring I/Q-imbalance ("no I/Q-comp."); (2) applying the compensation in Section 3 with perfect parameter estimation ("Perf. est."); (3) without impairments and assuming perfect channel estimation ("No imp."). In the

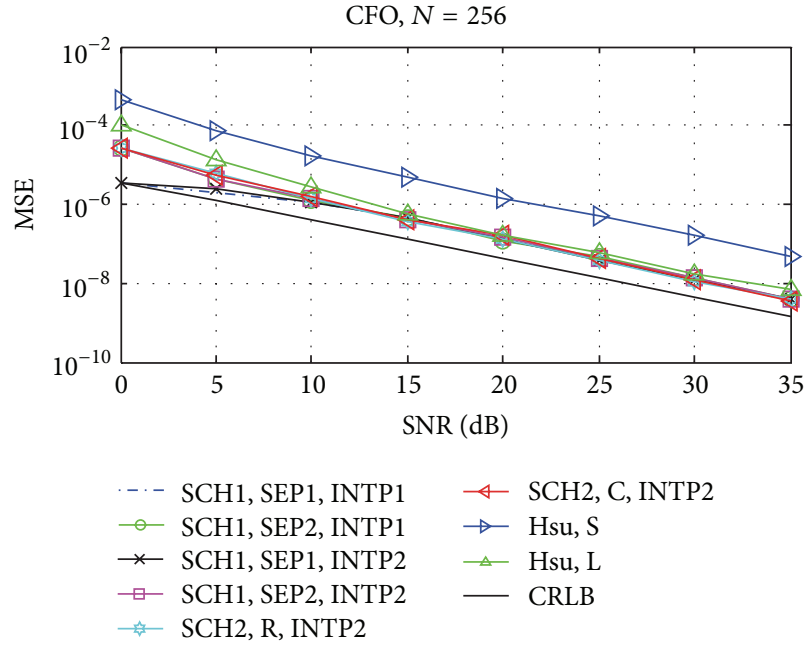

(a)

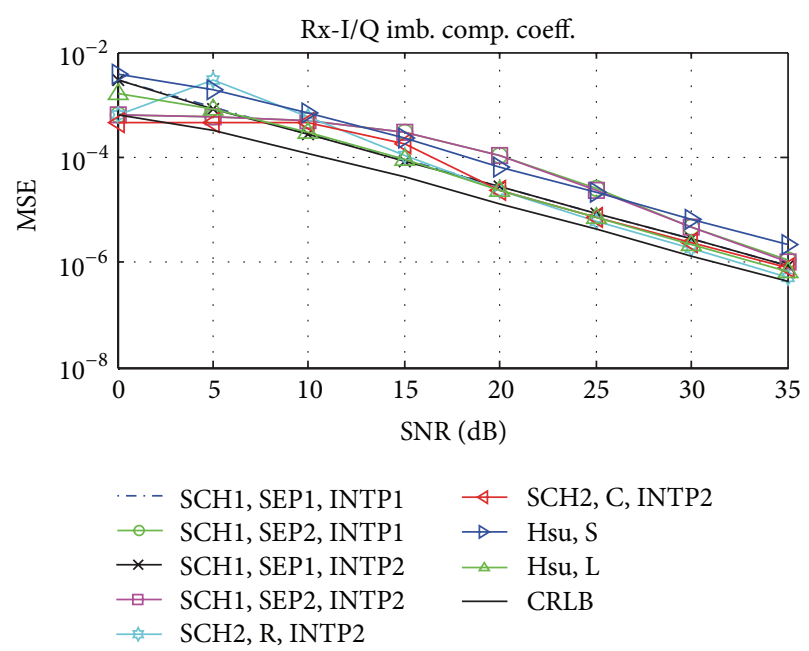

(b)

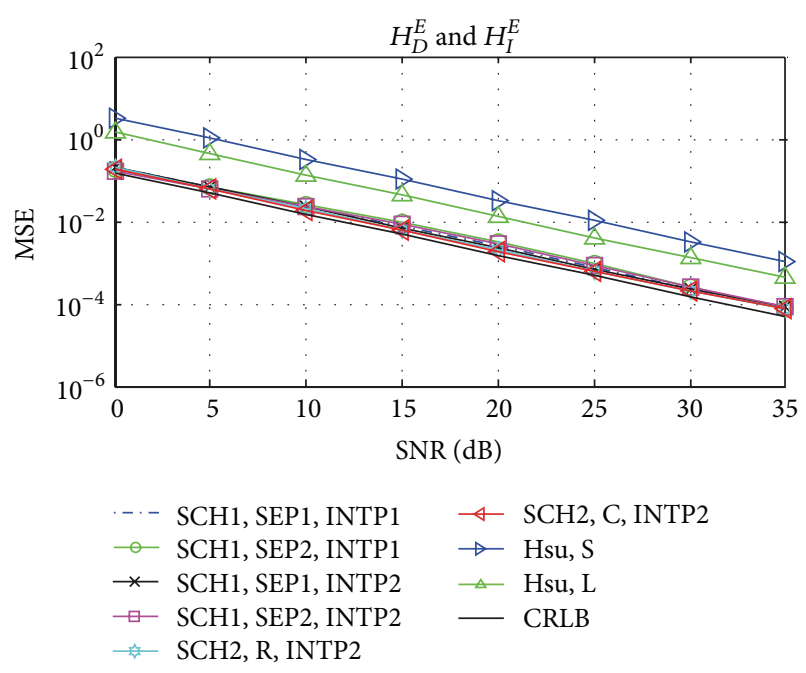

(c)

FIGURE 7: Estimation MSE as a function of SNR, $N_{I}=1, N=$ 256, and $\epsilon=0.25$. 


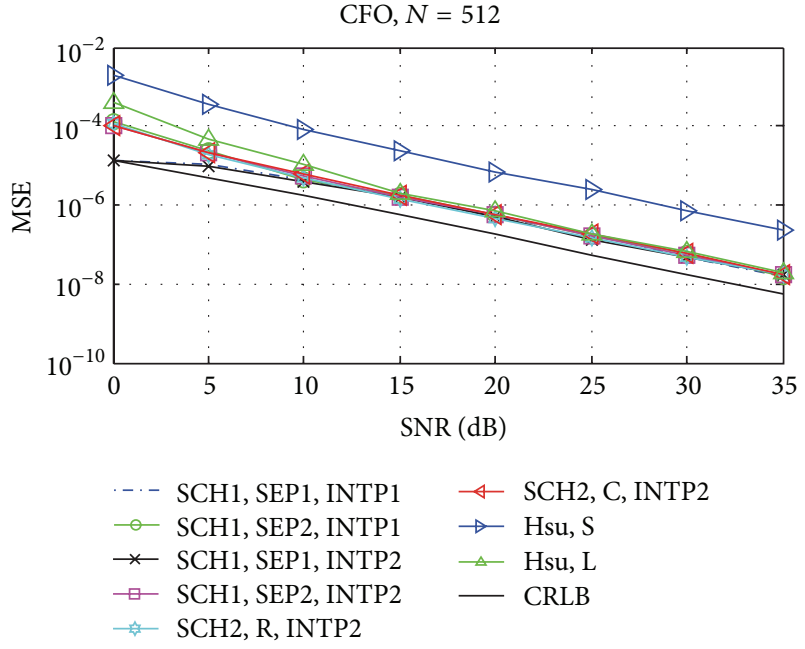

(a)
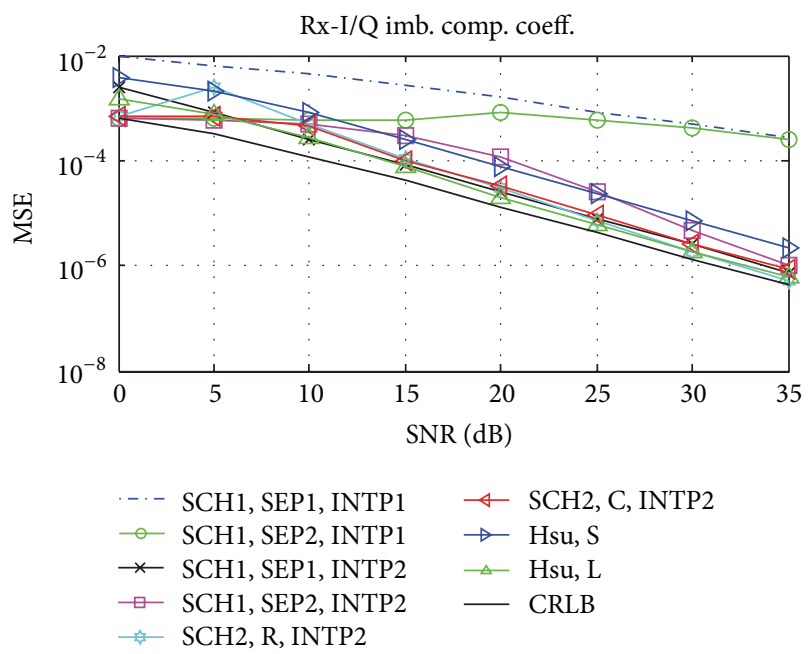

(b)
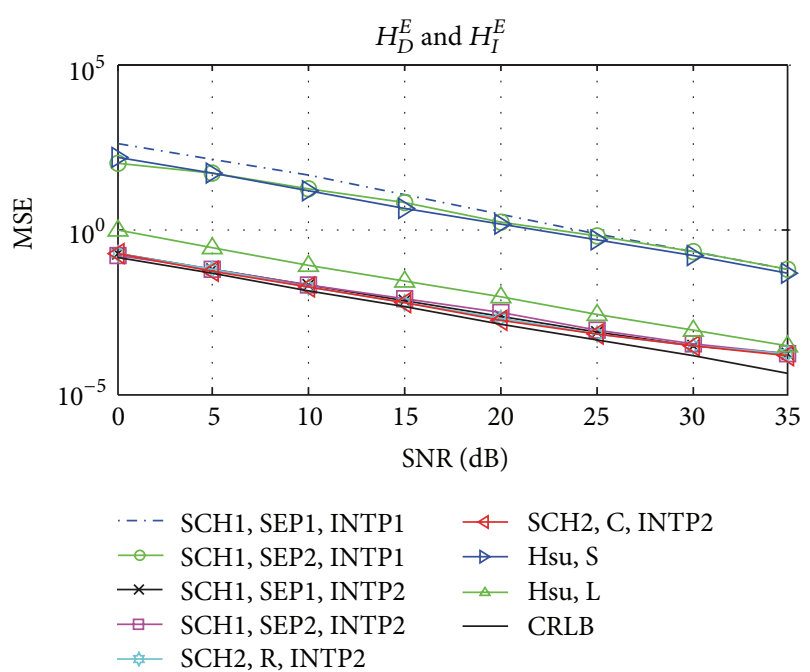

(c)

Figure 8: Estimation MSE as a function of SNR, $N_{I}=1, \quad N=$ 512 , and $\epsilon=0.5$.

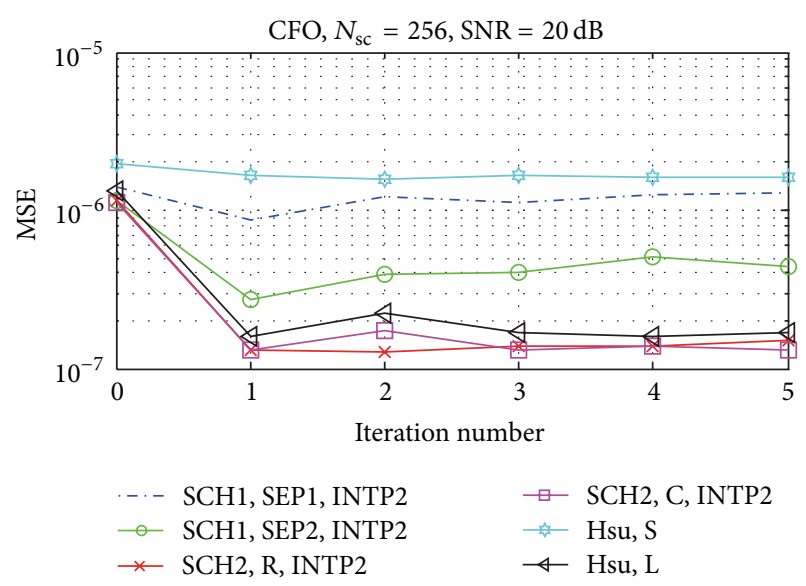

(a)

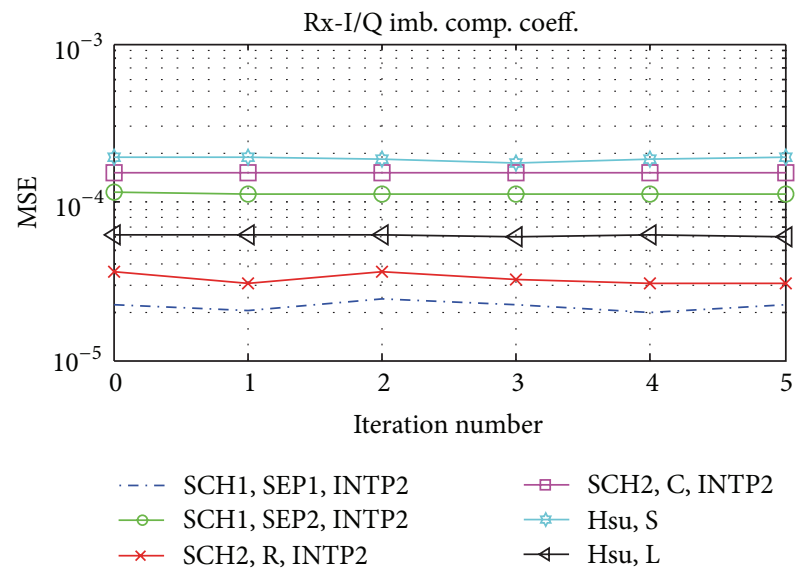

(b)

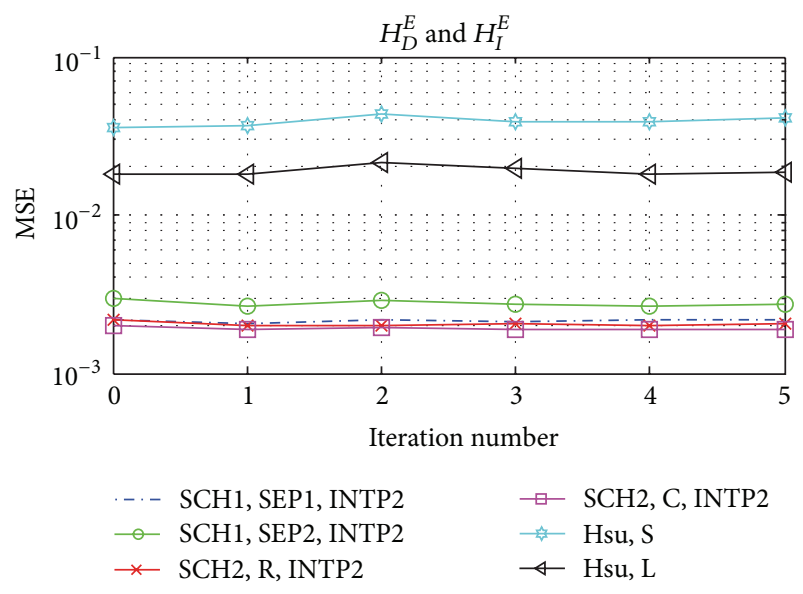

(c)

FIGURE 9: Estimation MSE as a function of iteration number, $N=$ 256, $\epsilon=0.15$, and $\mathrm{SNR}=20 \mathrm{~dB}$.

simulations, the $\mathrm{CFO}$ value was evenly distributed between 0 and $0.45 \mathrm{~N} / \mathrm{N}_{P}$. To avoid influence of residual $\mathrm{CFO}$, only 8 OFDM symbols are transmitted within each signal frame. (This is just a simulation example to investigate the performance without the need of phase tracking.) The used modulation scheme was 16-QAM. Moreover, subcarrierwise 


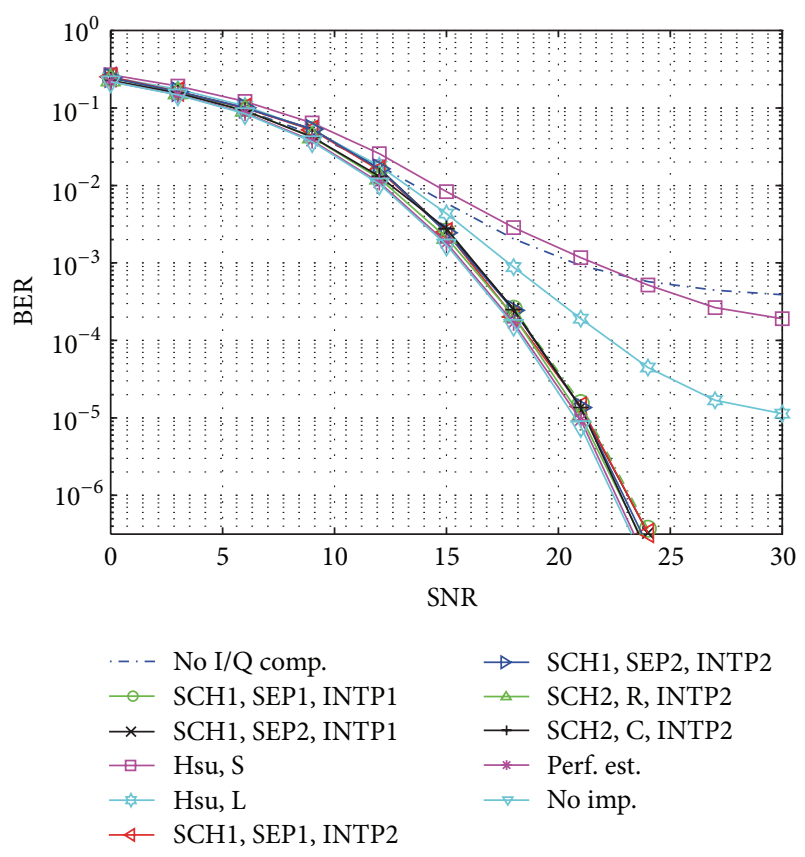

FIGURE 10: BER performance as a function of SNR, $N=$ 256 and $N_{0}=17$.

space time code (STC) and zero-forcing equalization (see Section 3) were applied. "Preamble-boosting" of $8 \mathrm{~dB}$ was applied for all schemes requiring parameter estimation. Figures 10 and 11 show the BER as a function of SNR for $N=256, N_{0}=17$ and $N=512, N_{0}=91$, respectively. As shown, provided perfect parameters, the performance with the compensation in Section 3 can be as good as that without impairments. We can also see that the SNR losses (due to parameter estimation error) of the proposed schemes with "INTP2" are relatively small (within $0.9 \mathrm{~dB}$ ). (There is only small performance difference between the proposed schemes with "INTP2"). Note that the longer the OFDM symbol, the more intercarrier interference and common phase error are caused by residual CFO. This may be the reason for the slightly higher SNR loss with $N=512, N_{0}=91$. In contrast to the proposed schemes, both the "Hsu S/L" schemes and the scheme ignoring I/Q-imbalance have error floors. While the latter scheme suffers from model mismatch, the scheme in [15] mainly suffers from large estimation error of $H_{\mathrm{I}}^{E\langle r, i\rangle}[k]$, for all $k$, which is resulted from a non-optimized preamble design.

Figure 12 shows the BER as a function of $\epsilon$ for both $N=$ 256, $N_{0}=17$ and $N=512, N_{0}=91$. As shown, these results comply with the MSE results in Figure 6. Slight BER increase can be observed at about $\epsilon N_{P} / N=0.05$ and $\epsilon N_{P} / N=0.5$. Note that at both $\epsilon=0$ and $\epsilon N_{P} / N=0.5$, the separate RxI/Q-imbalance compensation is deactivated (since separate estimation of the corresponding coefficients is impossible, see Sections 4.2 and 5.2). For $\epsilon=0, \mathrm{Rx}-\mathrm{I} / \mathrm{Q}$-imbalance can be comodeled by $H_{\mathrm{D} ; \mathrm{I}}^{E}$. Thus, no model mismatch is present, and no BER increase is observed. In contrast, $\epsilon N_{P} / N=0.5$, $\mathrm{Rx}$-I/Q-imbalance cannot be co-modeled by $H_{\mathrm{D} ; \mathrm{I}}^{E}$ due to the

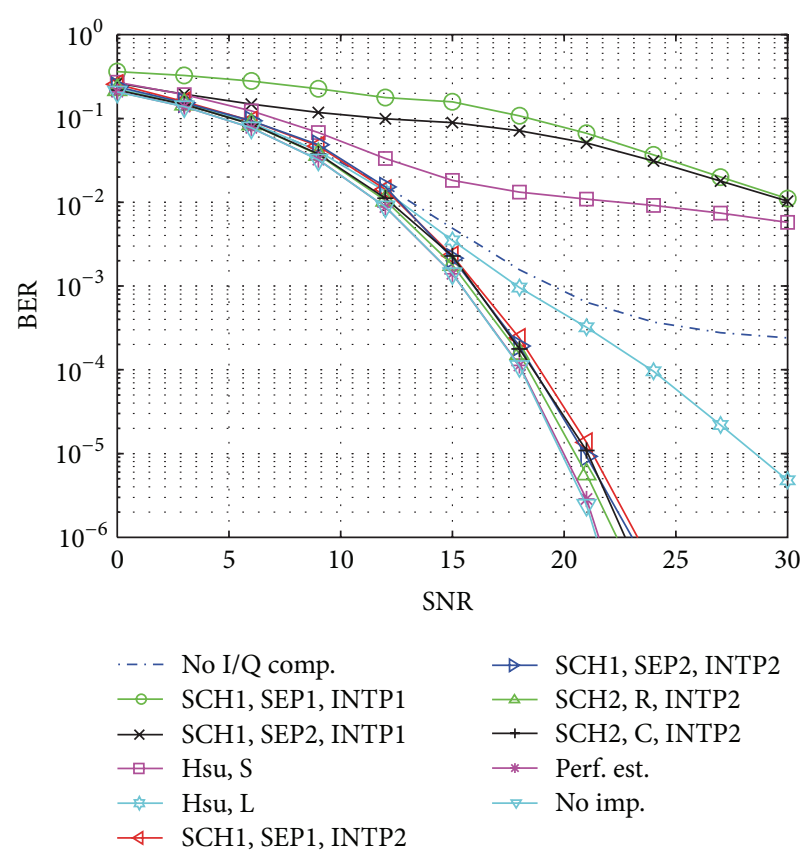

FIGURE 11: BER performance as a function of SNR, $N=$ 512 and $N_{0}=91$.

CFO influence. The corresponding model mismatch results in considerable BER increase. Thus, as shown in Figure 12, the BER at $\epsilon=0$ is much lower than that at $\epsilon N_{P} / N=0.5$.

\section{Computational Complexity Issues}

In this section, the computational complexity issue is addressed. The computational complexity of the proposed schemes can be divided into three parts: (1) parameter estimation; (2) equalization matrix calculation; and (3) the actual compensation. For part (2), the equalization matrix is calculated from the matrix equation, which is obtained based on (14).

7.1. Computational Complexity of Parameter Estimation. Table 3 shows the computational complexity expressions of different schemes in number of real multiplications (MULs). By applying the simulation parameters, Figure 13 can be obtained, which shows the number of required real MULs as a function of $N_{I}$ for different schemes. As shown, the "Hsu" schemes have the highest computational complexity. The main reason is the inefficient calculation of $H_{\mathrm{D} ; \mathrm{I}}^{E}$ (see $[11,15])$. Furthermore, "SCH1" has much lower computational complexity than "SCH2." The main reason was that the pseudoinverse computation in (43) of "SCH2, R" and that in (A.3) of "SCH2, C" are quite costly. Compared to "SCH2, C", "SCH2, R" has lower computational complexity, since realvalued computation is applied which considerably eased the pseudoinverse computation in (43).

7.2. Computational Complexity of Equalization Matrix Calculation and the Actual Compensation. Tables 4 and 5 


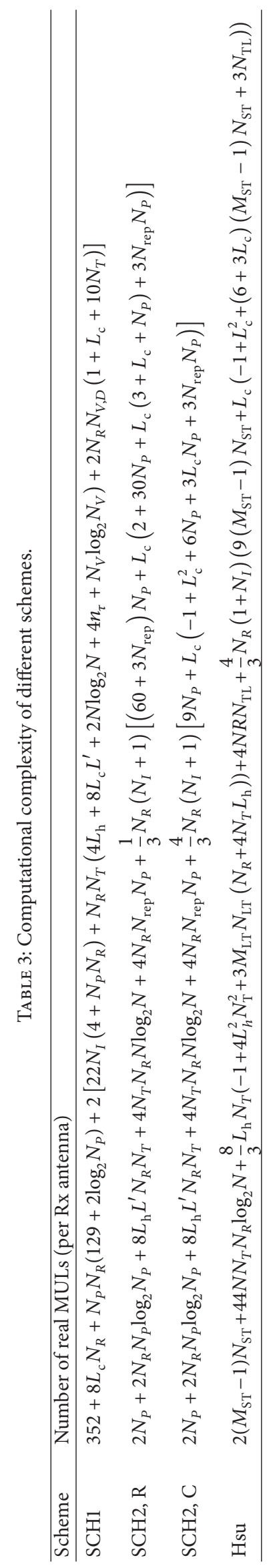




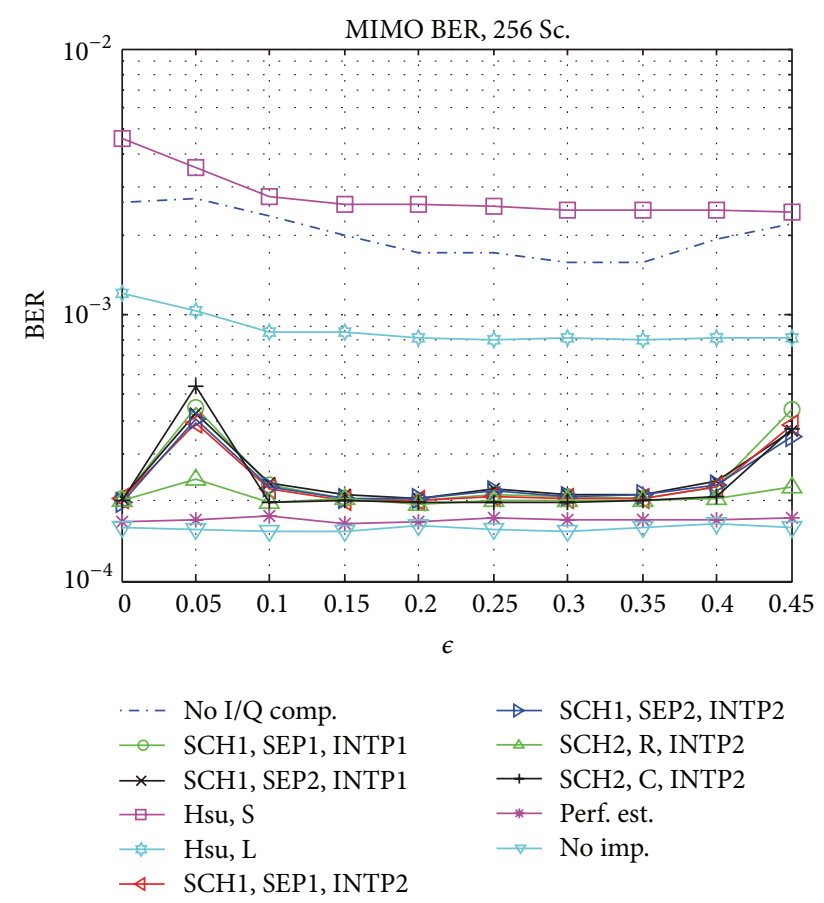

(a)

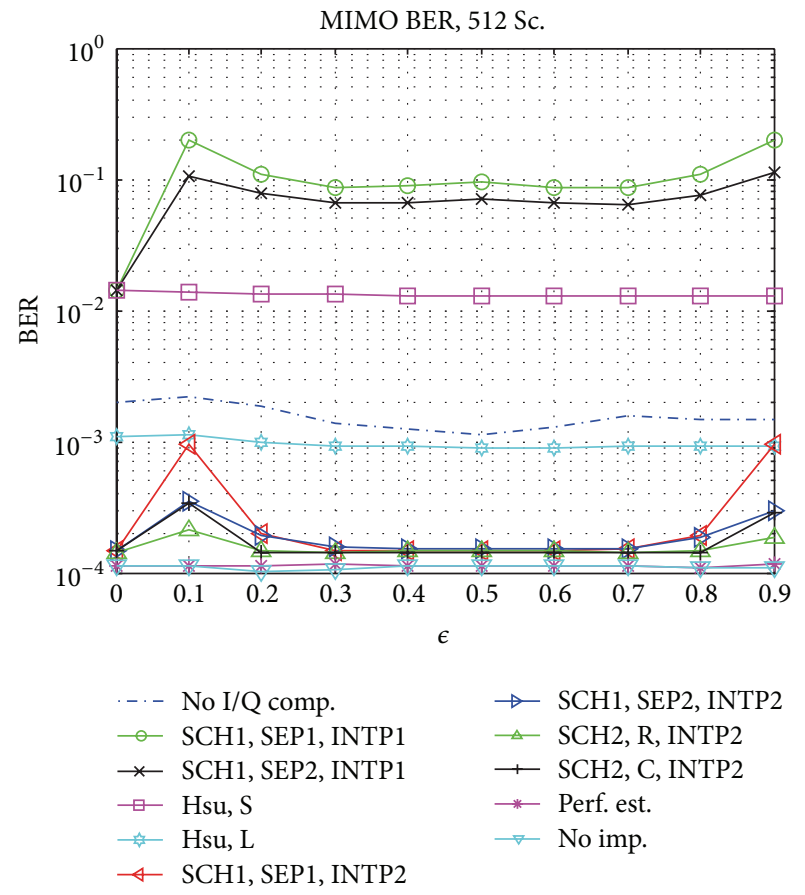

(b)

FIgURE 12: BER performance as a function of CFO. (a) $N=$ 256 and $N_{0}=17$; (b) $N=512$, and $N_{0}=91 ; \mathrm{SNR}=18 \mathrm{~dB}$.

summarize the computational complexity expressions of two different approaches for the calculation of equalization matrices and for the actual compensation, respectively. Both approaches only differ in the compensation method of the Tx-I/Q-imbalance and MIMO channel. The first approach

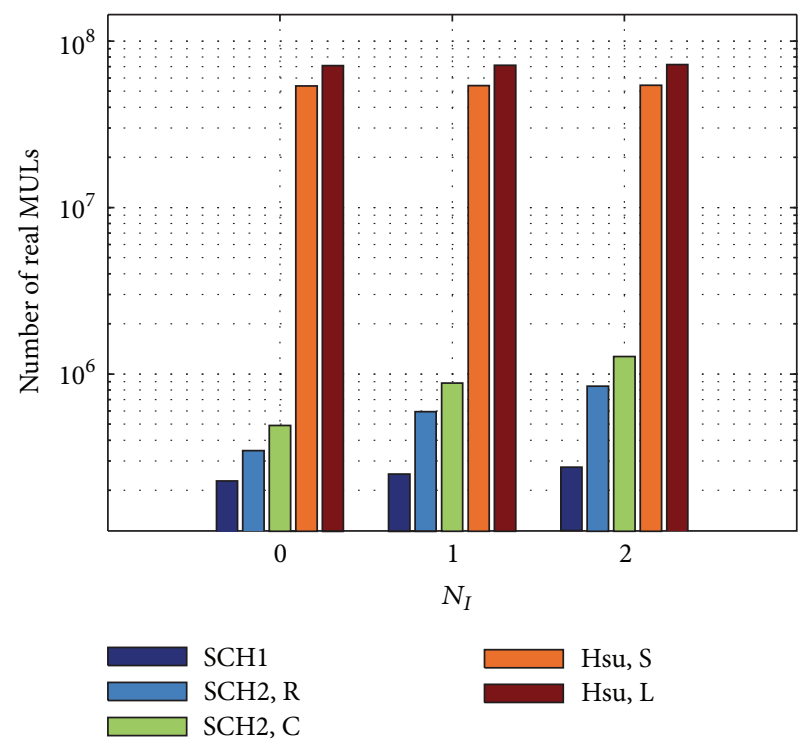

FIGURE 13: Number of required real MULs for different schemes as a function of $N_{I}, N=256$ and $N_{0}=17$.

applies the joint Tx-I/Q-imbalance and MIMO channel compensation in Section 3 (indicated by "Joint Tx IQ+Ch.") (the corresponding matrix equations can be found in [19]), while the second approach applies the separate Tx-I/Q-imbalance and MIMO channel compensation in [11] (indicated by "Sep. Tx IQ+Ch."). In [11], general MIMO structures of linear dispersion (LD) codes are considered, with STC and spacial multiplexing as special cases. It was assumed that at each subcarrier, $n_{s}$ data symbols are encoded in $\kappa$ consecutive OFDM symbols slots over $N_{T}$ Tx antennas. Furthermore, in this approach, the Tx-I/Q-imbalance is conducted subcarrierwise in frequency domain. For these two approaches, both real- and complex-valued FIR filters are compared for the compensation of Rx-I/Q-imbalance (indicated by " $R$ " and "C", resp.). The CFO compensation is done as described in Section 3. For simplicity, only zeros-forcing equalization and STC are considered.

With the simulation parameters, the complexity comparison in Figures 14 and 15 can be obtained (for approach (2), we have $n_{s}=2$ and $\kappa=2$ ). As shown in Figure 14, the "Sep. Tx IQ+Ch." scheme requires the highest computational complexity for equalization matrix calculation. The reason is that the subcarrierwise calculation of the Tx-I/Q-imbalance compensation coefficients in [11] is quite costly. Complexity reduction can be achieved by selecting just a subset of subcarriers where such coefficients are calculated. Afterwards, interpolation should be applied. Compared to the "Sep. Tx $\mathrm{IQ}+\mathrm{Ch}$." approach, the "Joint Tx IQ+Ch" approach has lower computational complexity.

Figure 15 shows that for the actual compensation of all approaches, using complex FIR filters for Rx-I/Q-imbalance compensation requires much higher complexity than using real-valued FIR filters. Furthermore, assuming the same filters for Rx-I/Q-imbalance compensation, the "Sep. Tx 


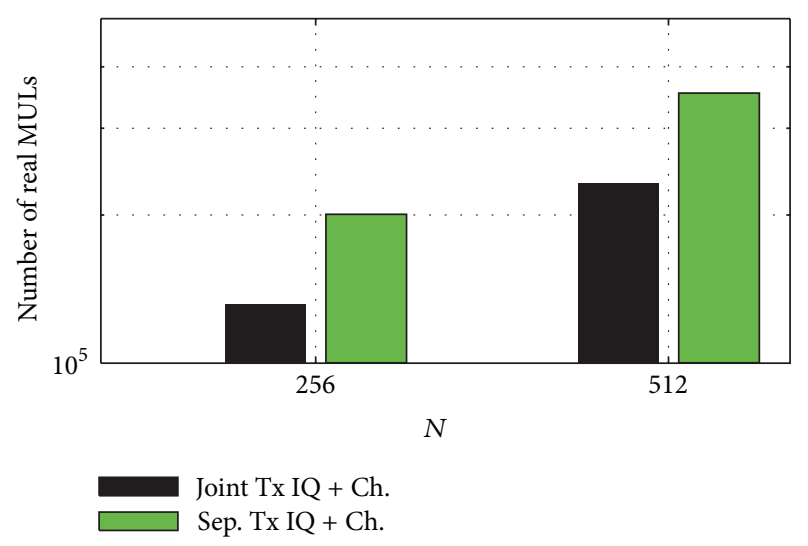

Figure 14: Computational complexity of different schemes for the calculation of the equalization matrix (for ZF signal equalization). STC is assumed.

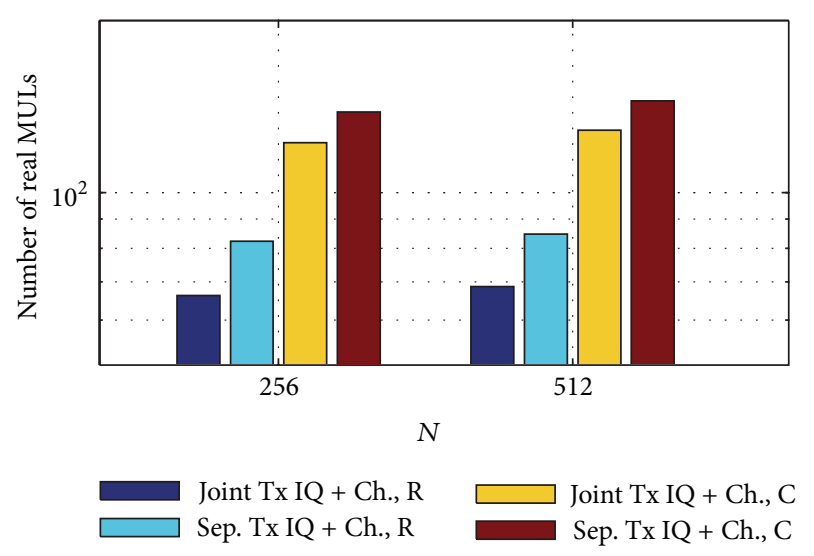

FIgURE 15: Computational complexity of different schemes for the actual compensation of CFO, I/Q-imbalance, and the MIMO channels. STC is assumed.

IQ+Ch." approach requires the highest computational complexity for the actual compensation. The complexity of the "Joint Tx IQ+Ch." approach is lower than that of the "Sep. Tx IQ+Ch." approach.

According to the results previous, it is more efficient to compensate Tx-I/Q imbalance jointly with the MIMO channel (when assuming zero-forcing equalization). Moreover, it is much more efficient to apply real-valued FIR filters to compensate for Rx-I/Q imbalance.

Finally, we can conclude that compared to the state-ofthe-art schemes, the proposed schemes require much lower computational complexity both for parameter estimation and impairment compensation.

\section{Conclusion}

In this paper, two preamble-based schemes are proposed for the joint estimation and compensation of CFO, Tx and $\mathrm{Rx}$ frequency-selective I/Q imbalance and the MIMO channel in OFDM systems. The first scheme applies three repetitions of a basic sequence as preamble and uses a closed-form CFO estimation. The second scheme applies two repetitions of the same basic sequence as preamble and uses iterative CFO and $\mathrm{Rx}-\mathrm{I} / \mathrm{Q}$-imbalance estimation. The design of the basic sequence is optimized to allow low preamble overhead and good estimation quality. The problem of critical CFO values is addressed by a soft switch method. Numerical simulation results have verified the effectiveness of the proposed schemes and show that better performance can be achieved than the state-of-the-art schemes. Furthermore, complexity analysis has shown that the proposed schemes have much lower computational complexity than the state-of-the-art schemes, allowing more efficient implementation. Among both proposed schemes, the first scheme has lower computational complexity, while the second scheme has lower preamble overhead. When both schemes apply the same preamble overhead, the second scheme can achieve slightly better performance. Based on the results above, we suggest to make the choice on the schemes according to the context and constraints of the system design (e.g., allowable overhead and computational complexity) as well as the advantages and disadvantages of these two candidate schemes.

An interesting future extension of the work in this paper may be to combine the bind Rx-I/Q-imbalance estimation scheme in [17] with preamble-based estimation of CFO, TxI/Q-imbalance, and the MIMO channel. The reason is that the bind scheme in [17] may provide better Rx-I/Q-imbalance estimation than preamble-based schemes due to exploitation of the noise.

\section{Appendix}

\section{Alternative Scheme for Joint CFO and Rx-I/Q Imbalance Estimation Using Complex-Valued Compensation Filters}

Except for the real-valued Rx-I/Q-imbalance compensation structure in Section 3, a complex-valued filter-based compensation can also be applied (as described in [15]). The $\mathrm{Rx}-$ I/Q-imbalance compensation using a complex-valued filter can be expressed as

$$
\hat{y}^{\langle r\rangle}[n]=y^{\langle r\rangle}[n]-y^{\langle r\rangle *}[n] * \rho^{C,\langle r\rangle}[n],
$$

where $\rho^{C}[n]$ denotes a length $L_{\mathrm{c}}$ complex-valued FIR filter with a dominant tap index $n_{\tau}$. Providing perfect Rx-I/Qimbalance compensation coefficients, we have the following relation, which is equivalent to (40):

$$
\left(\dot{\mathbf{y}}_{1}^{\langle r\rangle}-\dot{\mathbb{V}}_{1}^{\langle r\rangle *} \boldsymbol{\rho}^{C,\langle r\rangle}\right) e^{j \Omega}=\dot{\mathbf{y}}_{2}^{\langle r\rangle}-\dot{\mathbb{Y}}_{2}^{\langle r\rangle *} \boldsymbol{\rho}^{C,\langle r\rangle},
$$

where $\boldsymbol{\rho}^{C,\langle r\rangle}=\left[\rho^{C,\langle r\rangle}[0], \ldots, \rho^{C,\langle r\rangle}\left[L_{c}-1\right]\right]^{T}$, and $\dot{\mathbb{V}}_{b}^{\langle r\rangle}$ is a $N_{P} \times L_{\mathrm{c}}$ Toeplitz matrix with the $n_{\tau}^{\text {th }}$ column equal to $\dot{\mathbf{y}}_{b}^{\langle r\rangle}$. 
TABLE 4: Computational complexity expressions for equalization matrix computation.

\begin{tabular}{lc}
\hline Approach & Number of real MULs \\
\hline Joint Tx IQ + Ch. & $\frac{4}{3} N_{D} N_{T}\left(-1+4\left(1+6 N_{R}\right) N_{T}^{2}\right)$ \\
Sep. Tx IQ + Ch. & $\frac{2}{3} N_{D} n_{s}\left(-2+12 \kappa^{2} N_{R}^{2}+8 n_{s}^{2}+3 \kappa N_{R}\left(16 n_{s}+N_{T}\right)\right)$ \\
\hline
\end{tabular}

TABLE 5: Computational complexity expressions for the actual compensation.

\begin{tabular}{lc}
\hline Approach & Number of real MULs (per data symbol) \\
\hline Joint Tx IQ + Ch., R & $\frac{\left(6+L_{c}\right) N_{R}\left(N_{\mathrm{CP}}+N\right)}{N_{D}}+8 N_{T} N_{R}$ \\
Joint Tx IQ + Ch., C & $\frac{\left(5+4 L_{\mathrm{c}}\right) N_{R}\left(N_{\mathrm{CP}}+N\right)}{N_{D}}+8 N_{T} N_{R}$ \\
Sep. Tx IQ + Ch., R & $\frac{\kappa N_{R}\left(\left(6+L_{\mathrm{c}}\right) N_{\mathrm{CP}}+4 N_{D}\left(\kappa N_{R}+n_{s}\right)+\left(6+L_{\mathrm{c}}\right) N\right)}{N_{D} n_{s}}$ \\
Sep. Tx IQ + Ch., C & $\frac{\kappa N_{R}\left(\left(5+4 L_{\mathrm{c}}\right) N_{\mathrm{CP}}+4 N_{D}\left(\kappa N_{R}+n_{s}\right)+\left(5+4 L_{\mathrm{c}}\right) N\right)}{N_{D} n_{s}}$ \\
\hline
\end{tabular}

Based on (A.2), if we have a temporary estimate of $\epsilon$, that is, $\Omega$, the filter $\rho^{C,\langle r\rangle}$ can be obtained by

$$
\begin{aligned}
\widehat{\boldsymbol{\rho}}^{C,\langle r\rangle} & =\mathbb{R}_{\epsilon}^{\langle r\rangle} \mathbb{\mathbb { T }}_{\epsilon}^{\langle r\rangle}, \\
\text { with } \mathbb{R}_{\epsilon}^{\langle r\rangle}: & :=\dot{\mathbb{V}}_{1}^{\langle r\rangle *} e^{j \Omega}-\dot{\mathbb{V}}_{2}^{\langle r\rangle *}, \\
\mathbb{T}_{\epsilon}^{\langle r\rangle} & :=\dot{\mathbf{y}}_{1}^{\langle r\rangle} e^{j \Omega}-\dot{\mathbf{y}}_{2}^{\langle r\rangle} .
\end{aligned}
$$

An alternative joint $\mathrm{CFO}$ and $\mathrm{Rx}-\mathrm{I} / \mathrm{Q}$-imbalance scheme can be obtained by replacing (43) with (A.3) in Section 5.2 (the other steps of the scheme in Section 5.2 remain unchanged).

\section{Abbreviations}

ADC: Analog-to-digital converter

AWGN: Additive white Gaussian noise

BER: Bit error rate

CAZAC: Constant amplitude zero auto correlation

CFO: Common frequency offset

CLFE: Closed form estimator

CP: $\quad$ Cyclic prefix

CRLB: Cramer-Rao lower bound

DAC: Digital-to-Analog Converter

DFT: Discrete fourier transform

FDS: Frequency domain separation

FIR: $\quad$ Finite impulse response

IDFT: Inverse discrete fourier transform

IRR: Image rejection ratio

LLS: $\quad$ Linear least square

LMMSE: Linear minimum mean square error

LPF: Low-pass filter

LSE: Least-square estimation

MIMO: Multiple input multiple output

MLE: Maximum likelihood estimation

MSE: Mean square error

MUL: Multiplication

Nr.: Number

NLS: Nonlinear least-square
OFDM: Orthogonal frequency division multiplexing

PTEQ: Per-tone-equalization

RF: Radio frequency

Rx: Receiver

SCH: Scheme

SEP: Separation

SISO: Single input single output

SNR: Signal-to-noise ratio

STC: $\quad$ space time code

Tx: Transmitter.

\section{Mathematical Notations}

$(\cdot)^{*}: \quad$ Complex conjugate

$(\cdot)^{T}: \quad$ Transpose

$(\cdot)^{H}: \quad$ Conjugate transpose

$(\cdot)^{\dagger}$ : $\quad$ Pseudoinverse

$\operatorname{sgn}(\cdot)$ : Sign/signum function

$\|\cdot\|_{F}:$ The Frobenius norm

$\lceil c\rceil: \quad$ The smallest integer greater than $c$

*: $\quad$ Convolution

$(\odot)$ If not specified, the estimate of a parameter or a parameter vector/matrix

$(\mathbf{A})_{m, n}$ : The $(m, n)$ th element of the matrix $\mathbf{A}$, where the row/column indexes can be negative valued

$\mathbf{A}_{(\mathscr{R}, \mathscr{C})}$ : A submatrix of $\mathbf{A}$ by eliminating the rows and columns that are not within index sets $\mathscr{R}$ and $\mathscr{C}$, respectively

$\mathbf{V}_{\mathscr{R}}$ : A subvector obtained from $\mathbf{V}$ by eliminating the elements that are not within $\mathscr{R}$

$\mathbf{F}^{N}: \quad N \times N$ Fourier transform matrix with elements

$\left(\mathbf{F}^{N}\right)_{k, n}=e^{-j(2 \pi k n / N)}$, for all $k=$ $-N / 2, \ldots, N / 2-1 ; n=0, \ldots, N-1$

$\mathbf{0}_{M \times N}:$ An $M \times N$ zero matrix 


\begin{tabular}{|c|c|}
\hline $\mathbf{I}_{N}:$ & An $N \times N$ identity matrix \\
\hline$-\mathscr{I}:$ & $\begin{array}{l}\text { Order-reversed and componentwise } \\
\text { negated version of the set } \mathscr{I}\end{array}$ \\
\hline $\mathscr{F}_{N}\{x[n]\}:$ & $\begin{array}{l}N \text {-point DFT: } X[k]=\mathscr{F}_{N}\{x[n]\}= \\
\sum_{n=0}^{N-1} x[n] e^{-j(2 \pi k n / N)}, \text { for all } k= \\
-N / 2, \ldots, N / 2-1\end{array}$ \\
\hline $\mathscr{F}_{N}^{-1}\{X[k]\}$ & $\begin{array}{l}N \text {-point IDFT: } x[n]=\mathscr{F}_{N}^{-1}\{X[k]\}= \\
(1 / N) \sum_{k=-N / 2}^{N / 2-1} X[k] e^{j(2 \pi k n / N)}, \text { for all } n= \\
0, \ldots, N-1\end{array}$ \\
\hline subscript re; im: & $\begin{array}{l}\text { Real/imaginary part, for example, } \\
s(t)=s_{\mathrm{re}}(t)+j s_{\mathrm{im}}(t)\end{array}$ \\
\hline$X^{\operatorname{Re}}[k]:$ & $\mathscr{F}_{N}\left\{x_{\text {re }}[n]\right\}$ \\
\hline $\begin{array}{l}X^{\operatorname{Im}}[k]: \\
\text { superscript }(i):\end{array}$ & $\begin{array}{l}\mathscr{F}_{N}\left\{x_{\mathrm{im}}[n]\right\} \\
i \text { th TX }\end{array}$ \\
\hline $\begin{array}{l}\text { superscript }\langle r\rangle: \\
\text { “;” in sub- } \\
\text { /superscripts: }\end{array}$ & $\begin{array}{l}r \text { th RX } \\
\text { "or," for example, } h_{\mathrm{D} ; \mathrm{I}} \text { indicates } h_{\mathrm{D}} \text { or } h_{\mathrm{I}}\end{array}$ \\
\hline$\delta[n]:$ & $\begin{array}{l}\text { Unit impulse, that } \\
\text { is, } \delta[0]=1, \delta[n]=0 \text {, for all } n \neq 0\end{array}$ \\
\hline$(\bullet):$ & Preamble-related signals \\
\hline & $(\bullet) e^{-j(2 \pi / N) \epsilon n}$ \\
\hline & $(\cdot) e^{j(2 \pi / N) e n}$ \\
\hline
\end{tabular}

\section{Authors' Contributions}

The major contribution of J. Luo has been made when he was affiliated with Fraunhofer Heinrich Hertz Institute in Berlin, Germany.

\section{References}

[1] B. Razavi, "Design considerations for direct-conversion receivers," IEEE Transactions on Circuits and Systems II, vol. 44, no. 6, pp. 428-435, 1997.

[2] G. Xing, M. Shen, and H. Liu, "Frequency offset and I/Q imbalance compensation for OFDM direct-conversion receivers," in Proceedings of the IEEE International Conference on Accoustics, Speech, and Signal Processing (ICASSP '03), pp. 708-711, April 2003.

[3] G. Gil, I. H. Sohn, Y. H. Lee, Y. I. Song, and J. K. Park, "Joint ML estimation of I/Q mismatch, DC offset, carrier frequency, and channel for direct-conversion receivers," in Proceedings of the 57th IEEE Semiannual Vehicular Technology Conference (VTC '03-Spring), vol. 4, pp. 2348-2352, Jeju, Korea, April 2003.

[4] J. Tubbax, A. Fort, L. V. der Perre et al., "Joint compensation of IQ imbalance and frequency Offset in OFDM systems," in Proceedings of the IEEE Global Telecommunications Conference (GLOBECOM '03), vol. 4, pp. 2365-2369, San Francisco, Calif, USA, December 2003.

[5] S. de Rore, E. Lopez-Estraviz, F. Horlin, and L. van der Perre, "Joint estimation of carrier frequency offset and IQ imbalance for $4 \mathrm{G}$ mobile wireless systems," in Proceedings of the IEEE International Conference on Communications (ICC '06), pp. 2066-2071, July 2006.

[6] H. Lin, X. Zhu, and K. Yamashita, "Pilot-aided low-complexity $\mathrm{CFO}$ and I/Q imbalance compensation for OFDM systems," in
Proceedings of the IEEE International Conference on Communications (ICC '08), pp. 713-717, May 2008.

[7] H. Lin, X. Zhu, and K. Yamashita, "Hybrid domain compensation for analog impairments in OFDM systems," in Proceedings of the IEEE Global Telecommunications Conference (GLOBECOM '08), New Orleans, La, USA, November 2008.

[8] J. Feigin and D. Brady, "Joint transmitter/receiver I/Q imbalance compensation for direct conversion OFDM in packet-switched multipath environments," IEEE Transactions on Signal Processing, vol. 57, no. 11, pp. 4588-4593, 2009.

[9] L. Lanante Jr., M. Kurosaki, and H. Ochi, "Low complexity compensation of frequency dependent I/Q imbalance and carrier frequency offset for direct conversion receivers," in Proceedings of the IEEE International Symposium on Circuits and Systems (ISCAS '10), pp. 2067-2070, Paris, France, June 2010.

[10] D. Tandur and M. Moonen, "Compensation of RF impairments in MIMO OFDM systems," in Proceedings of the IEEE International Conference on Acoustics, Speech, and Signal Processing (ICASSP '08), Las Vegas, Nev, USA, March 2008.

[11] C. J. Hsu and W. H. Sheen, "Joint estimation and compensation of transmitter and receiver radio impairments in MIMOOFDM receivers," in Proceedings of the IEEE 20th International Symposium on Personal, Indoor and Mobile Radio Communications (PIMRC '09), September 2009.

[12] C. J. Hsu, R. Cheng, and W. H. Sheen, "Joint least squares estimation of frequency, DC offset, I-Q imbalance, and channel in MIMO receivers," IEEE Transactions on Vehicular Technology, vol. 58, no. 5, pp. 2201-2213, 2009.

[13] J. Gao, X. Zhu, H. Lin, and A. K. Nandi, "Kalman filtering based compensation for I/Q imbalance and CFO in time-varying MIMO OFDM systems," in Proceedings of the IEEE Global Telecommunications Conference (GLOBECOM '09), November 2009.

[14] Y. H. Chung and S. M. Phoong, "Joint estimation of I/Q imbalance, CFO and channel response for MIMO OFDM systems," IEEE Transactions on Communications, vol. 58, no. 5, pp. 1485-1492, 2010.

[15] C. J. Hsu and W. H. Sheen, "Compensation of cascaded radio impairments in MIMO-OFDM systems with direct-conversion architecture," in Proceedings of the Global Mobile Congress (GMC '10), October 2010.

[16] J. Luo, W. Keusgen, and A. Kortke, "Preamble based joint CFO, frequency-selective I/Q-imbalance and channel estimation and compensation in MIMO OFDM systems," in Proceedings of the IEEE Vehicular Technology Society, Conference (VTC '11-Fall), March 2011.

[17] L. Anttila, M. Valkama, and M. Renfors, "Circularity-based I/Q imbalance compensation in wideband direct-conversion receivers," IEEE Transactions on Vehicular Technology, vol. 57, no. 4, pp. 2099-2113, 2008.

[18] A. Kiayani, L. Anttila, Y. Zou, and M. Valkama, "Advanced receiver design for mitigating multiple $\mathrm{RF}$ impairments in OFDM systems: algorithms and RF measurements," Journal of Electrical and Computer Engineering, vol. 2012, Article ID 730537, 16 pages, 2012. 
[19] J. Luo, W. Keusgen, and A. Kortke, "Preamble designs for effcient joint channel and frequency-selective I/Q-imbalance compensation in MIMO OFDM systems," in Proceedings of the Wireless Communications and Networking Conference (WCNC '10), pp. 1-6, April 2010.

[20] M. Peter, W. Keusgen, and A. Kortke, "Measurement and analysis of the $60 \mathrm{GHz}$ in-vehicular broadband radio channel," in Proceedings of the IEEE Vehicular Technology Society, Conference (VTC '07-Fall), Baltimore, Md, USA, 2007. 

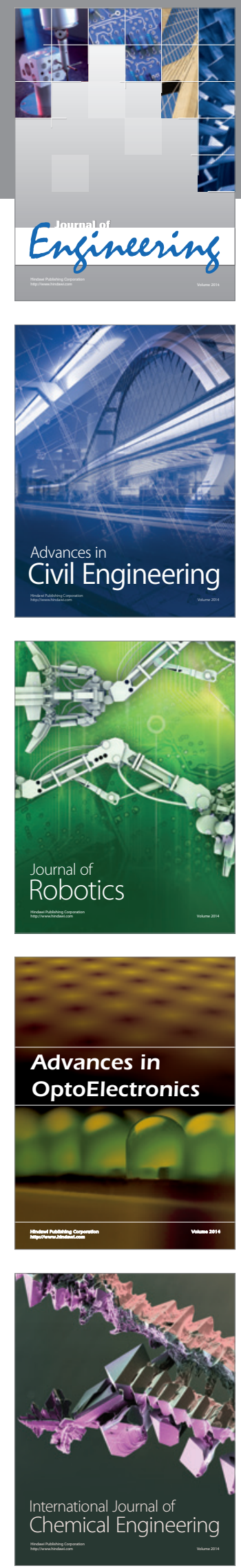

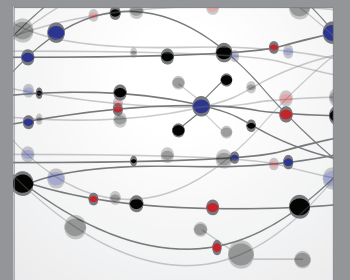

The Scientific World Journal
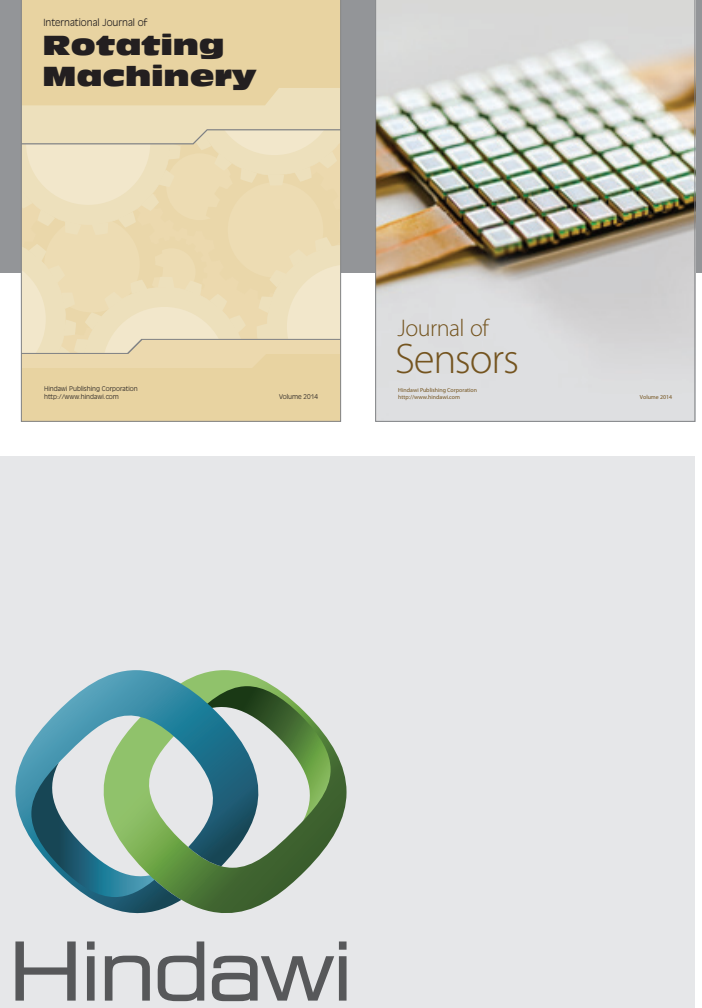

Submit your manuscripts at http://www.hindawi.com
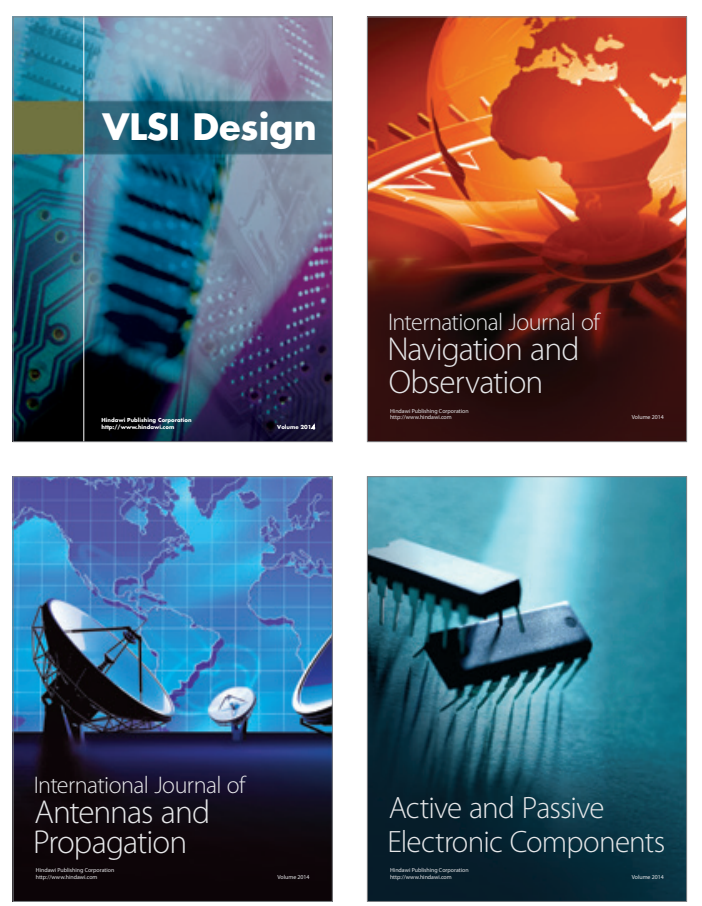
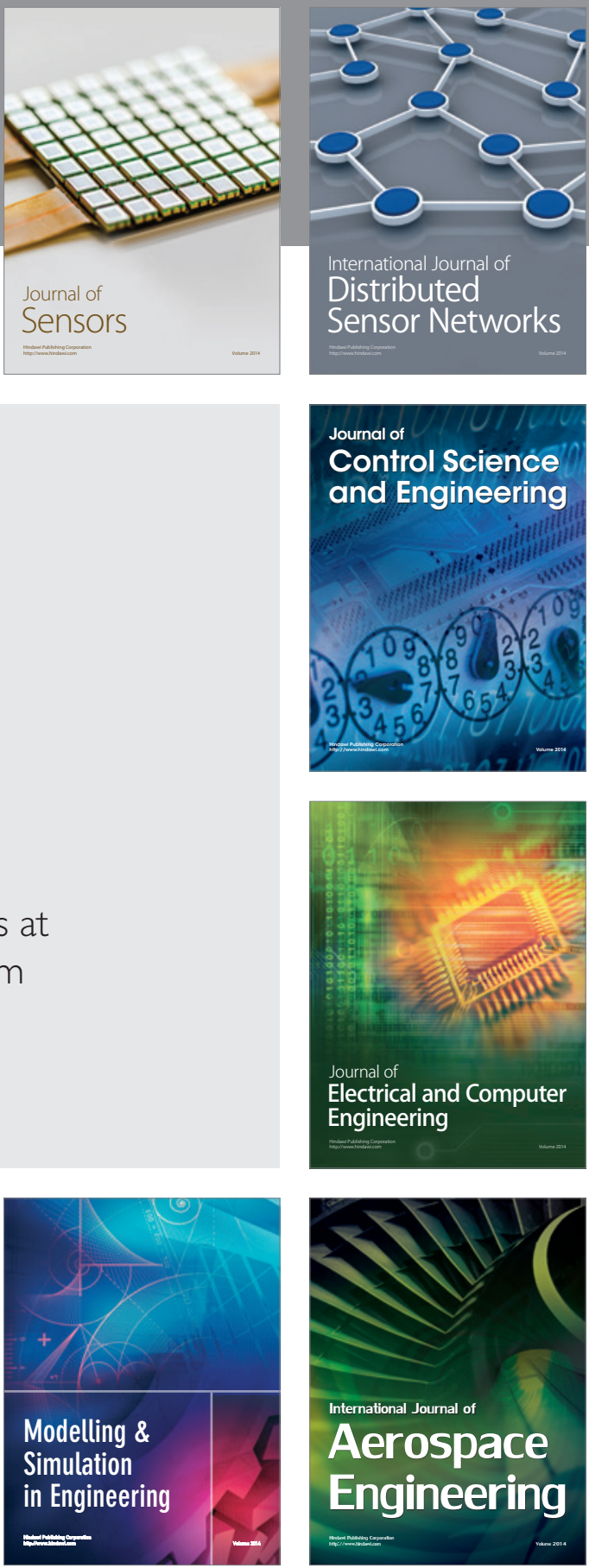

Journal of

Control Science

and Engineering
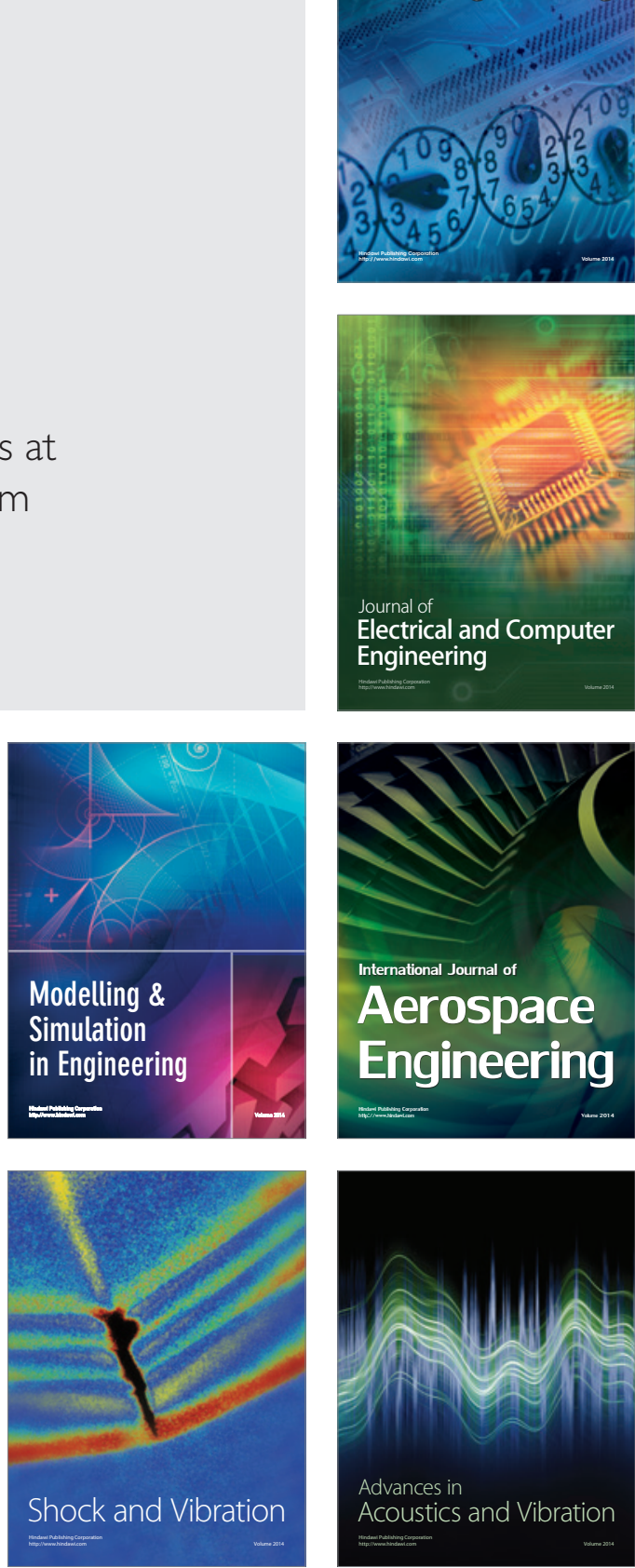